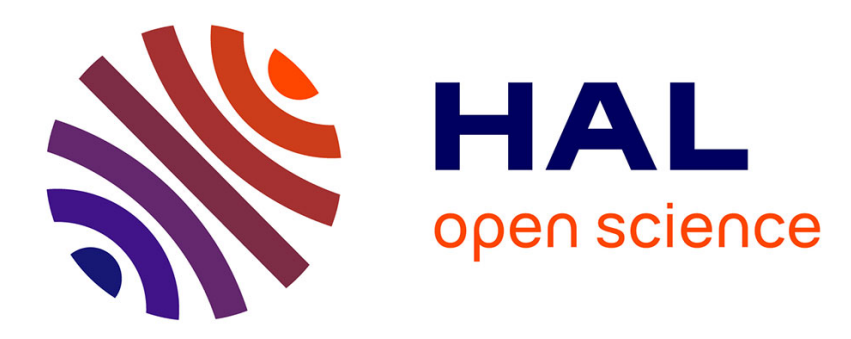

\title{
Multiattribute preference models with reference points
}

Denis Bouyssou, Thierry Marchant

\section{To cite this version:}

Denis Bouyssou, Thierry Marchant. Multiattribute preference models with reference points. 2011. hal-00606942

\section{HAL Id: hal-00606942 \\ https://hal.science/hal-00606942}

Preprint submitted on 7 Jul 2011

HAL is a multi-disciplinary open access archive for the deposit and dissemination of scientific research documents, whether they are published or not. The documents may come from teaching and research institutions in France or abroad, or from public or private research centers.
L'archive ouverte pluridisciplinaire HAL, est destinée au dépôt et à la diffusion de documents scientifiques de niveau recherche, publiés ou non, émanant des établissements d'enseignement et de recherche français ou étrangers, des laboratoires publics ou privés. 


\title{
Multiattribute preference models with reference points ${ }^{\text {th }}$
}

\author{
Denis Bouyssou ${ }^{\mathrm{a}, \mathrm{b}}$, Thierry Marchant ${ }^{\mathrm{c}}$ \\ ${ }^{a}$ CNRS-LAMSADE, UMR7243, F-75775 Paris Cedex 16, France \\ ${ }^{b}$ Université Paris Dauphine, F-75775 Paris Cedex 16, France \\ ${ }^{c}$ Ghent University, H. Dunantlaan, 1, B-9000 Gent, Belgium
}

\begin{abstract}
In the context of multiple attribute decision making, preference models making use of reference points in an ordinal way have recently been introduced in the literature. This text proposes an axiomatic analysis of such models, with a particular emphasis on the case in which there is only one reference point. Our analysis uses a general conjoint measurement model resting on the study of traces induced on attributes by the preference relation and using conditions guaranteeing that these traces are complete. Models using reference points are shown to be a particular case of this general model. The number of reference points is linked to the number of equivalence classes distinguished by the traces. When there is only one reference point, the induced traces are quite rough, distinguishing at most two distinct equivalence classes. We study the relation between the model using a single reference point and other preference models proposed in the literature.

Keywords: Multiple Criteria Decision Making Reference point Conjoint Measurement
\end{abstract}

\section{Introduction}

In a series of papers, Rolland (2003, 2006a,b, 2008, 2011) (see also Perny and Rolland 2006, in the related context of decision making under uncertainty) has suggested to use reference points ${ }^{1}$ in an ordinal way to build

\footnotetext{
Authors are listed alphabetically. They have equally contributed to this paper. We wish to thank Patrice Perny and Antoine Rolland for their very helpful comments on an earlier draft of this text. The usual caveat applies.

Email addresses: bouyssou@lamsade.dauphine.fr (Denis Bouyssou), thierry.marchant@UGent.be (Thierry Marchant)
} 
preference models for multiattributed alternatives. This idea can be traced back to Fargier and Perny (1999) and Dubois, Fargier, and Perny (2003, p. $247)$. In these models, the preference between alternatives $x$ and $y$ rests on a comparison in terms of "importance" of the sets of attributes for which $x$ and $y$ are above the reference points. Rolland has analyzed the interest of such models and has proposed axioms that could characterize them. Most of his axiomatic analysis supposes that the reference points are known beforehand ${ }^{2}$. Including reference points in the primitives of the model is a strong hypothesis and raises observational questions. Moreover, he invokes conditions that seem to be quite specific to models using reference points. It is therefore not easy to use them in order to compare these models with other ones that have been proposed and characterized in the literature.

The aim of this text is to propose an axiomatic analysis of preference models with reference points using the traditional primitives of conjoint measurement, i.e., a preference relation on the set of alternatives. Our analysis uses a general conjoint measurement model resting on the study of traces induced on attributes by the preference relation and using conditions guaranteeing that these traces are complete. We show that preference models with reference points are a particular case of this general model. This will allow us to characterize preference models with reference points using conditions that will facilitate their comparison with other preference models proposed in the literature. We concentrate on preference models that use a single reference point. These models induce traces on each attribute that are quite rough, distinguishing at most two distinct equivalence classes. These models are then compared with other types of preference models introduced in the literature, most notably, with models based on a discrete Sugeno integral (Bouyssou, Marchant, and Pirlot 2009, Greco, Matarazzo, and Słowiński 2004) and models based on the notion of concordance (Bouyssou and Pirlot 2005b, 2007).

Our general strategy will be similar to the one used in Bouyssou and Pir-

\footnotetext{
${ }^{1}$ The notion of "reference point" is unfortunately used in the literature with many different meanings. The interpretation of the reference points in the models studied in this paper is discussed below. These reference points have little to do with the reference point used in prospect theory to distinguish gains from losses (Kahneman and Tversky 1979, Tversky and Kahneman 1992) or from the reference points used as a crucial element in the framing of decisions (Tversky and Kahneman 1986).

${ }^{2}$ For exceptions, see Rolland (2003, Th. 3) and Rolland (2008, Section 2.1.2).
} 
lot $(2005 \mathrm{~b}, 2007)$ to analyze models based on the notion of concordance. They have shown that such models could be seen as particular cases of the general conjoint measurement models developed in Bouyssou and Pirlot $(2002,2004 a)$ that generate complete traces on differences between levels in which these traces are "rough", i.e., only distinguishing a limited number of equivalence classes. We show here that models using reference points are a particular case of models inducing complete traces on levels developed in Bouyssou and Pirlot (2004b) in which these traces are "rough" (for a general overview of preference models based on different kinds of traces, we refer to Bouyssou and Pirlot 2005a).

The paper is organized as follows. Section 2 introduces our notation and setting. Section 3 formalizes preference models using a single reference point. Section 4 recalls the main ingredients of the general conjoint measurement models introduced in Bouyssou and Pirlot (2004b). Section 5 characterizes preference models using a single reference point. Section 6 studies the relations between concordance relations and preference models using a single reference point. Section 7 is devoted to the study of preference models using a single reference point that are weak orders. A final section discusses our findings. Appendix D extends our results to preference models using several reference points.

\section{Background}

\subsection{Binary relations}

A binary relation $\mathcal{K}$ on a set $A$ is a subset of $A \times A$. We often write $a \mathcal{K} b$ instead of $(a, b) \in \mathcal{K}$. The symmetric (resp. asymmetric) part of $\mathcal{K}$ is the binary relation $\sigma(\mathcal{K})($ resp. $\alpha(\mathcal{K}))$ on $A$ such that $a \sigma(\mathcal{K}) b$ iff $[a \mathcal{K} b$ and $b \mathcal{K} a](\operatorname{resp} . a \alpha(\mathcal{K}) b$ iff $[a \mathcal{K} b$ and $\operatorname{Not}[b \mathcal{K} a]])$.

An equivalence is a reflexive $(a \mathcal{K} a)$, symmetric $(a \mathcal{K} b \Rightarrow b \mathcal{K} a)$ and transitive $([a \mathcal{K} b$ and $b \mathcal{K} c] \Rightarrow a \mathcal{K} c)$ binary relation on $A$. An equivalence relation partitions $A$ into equivalence classes. The set of equivalence classes induced by the equivalence $\mathcal{K}$ is denoted by $A / \mathcal{K}$.

A weak order is a complete $(a \mathcal{K} b$ or $b \mathcal{K} a)$ and transitive binary relation. When $\mathcal{K}$ is a weak order on $A$, it is clear that $\sigma(\mathcal{K})$ is an equivalence. We often abuse terminology and speak of equivalence classes of the weak order $\mathcal{K}$ instead of the equivalence classes of $\sigma(\mathcal{K})$. In this case we also speak of the first, second, ..., last equivalence class of $\mathcal{K}$. 
A semiorder is a reflexive $(a \mathcal{K} a)$, Ferrers $([a \mathcal{K} b$ and $c \mathcal{K} d]$ imply $[a \mathcal{K} d$ or $c \mathcal{K} b])$ and semitransitive $([a \mathcal{K} b$ and $b \mathcal{K} c]$ imply $[a \mathcal{K} d$ or $d \mathcal{K} c]$ ) binary relation. If $\mathcal{K}$ is a semiorder, it is well known (Aleskerov, Bouyssou, and Monjardet 2007) that the relation $\mathcal{K}^{\circ}$ defined letting, for all $a, b, c \in A$,

$$
a \mathcal{K}^{\circ} b \Leftrightarrow\left\{\begin{array}{l}
b \mathcal{K} c \Rightarrow a \mathcal{K} c, \\
c \mathcal{K} a \Rightarrow c \mathcal{K} b
\end{array}\right.
$$

is a weak order.

\subsection{Notation}

In this paper $\succsim$ will always denote a binary relation on a set $X=\prod_{i=1}^{n} X_{i}$ with $n \geq 2$. Elements of $X$ will be interpreted as alternatives evaluated on a set $N=\{1,2, \ldots, n\}$ of attributes and $\succsim$ as an "at least as good as" relation between these alternatives. We denote by $\succ$ (resp. $\sim)$ the asymmetric (resp. symmetric) part of $\succsim$. A similar convention holds when $\succsim$ is starred, superscripted and/or subscripted.

For any nonempty subset $J$ of the set of attributes $N$, we denote by $X_{J}$ (resp. $\left.X_{-J}\right)$ the set $\prod_{i \in J} X_{i}$ (resp. $\prod_{i \in N \backslash J} X_{i}$ ). When $x, y \in X$, with customary abuse of notation, $\left(x_{J}, y_{-J}\right)$ will denote the element $w \in X$ such that $w_{i}=x_{i}$ if $i \in J$ and $w_{i}=y_{i}$ otherwise. We sometimes omit braces around sets. For instance, when $J=\{i\}$ we write $X_{-i}$ and $\left(x_{i}, y_{-i}\right)$.

We say that attribute $i \in N$ is influential (for $\succsim$ ) if there are $x_{i}, y_{i}, z_{i}, w_{i} \in$ $X_{i}$ and $a_{-i}, b_{-i} \in X_{-i}$ such that $\left(x_{i}, a_{-i}\right) \succsim\left(y_{i}, b_{-i}\right)$ and $\left(z_{i}, a_{-i}\right) \succsim\left(w_{i}, b_{-i}\right)$ and degenerate otherwise. A degenerate attribute has no influence whatsoever on the comparison of the elements of $X$ and may be suppressed from $N$. As in Bouyssou and Pirlot (2005b), in order to avoid unnecessary minor complications, we suppose henceforth that all attributes in $N$ are influential.

Let $J \subset N$ be a proper nonempty subset of attributes. We say that $\succsim$ is independent for $J$ if, for all $x_{J}, y_{J} \in X_{J}$,

$$
\begin{gathered}
\left(x_{J}, z_{-J}\right) \succsim\left(y_{J}, z_{-J}\right), \text { for some } z_{-J} \in X_{-J} \Rightarrow \\
\left(x_{J}, w_{-J}\right) \succsim\left(y_{J}, w_{-J}\right), \text { for all } w_{-J} \in X_{-J} .
\end{gathered}
$$

If $\succsim$ is independent for all proper nonempty subsets of $N$, we say that $\succsim$ is independent. It is clear that $\succsim$ is independent iff $\succsim$ is independent for $N \backslash\{i\}$, for all $i \in N$. 


\section{Preference models with a single reference point}

The model that we study was introduced by Rolland (2003, 2006a,b, 2008,?). It has close connections with ELECTRE TRI (Roy and Bouyssou 1993, Chap. 6). Remember that ELECTRE TRI is a technique used to assign alternatives to ordered categories. Suppose that there are only two categories: $\mathscr{A}$ and $\mathscr{U}, \mathscr{A}$ being the best category. The limit between these two categories is indicated by a profile $p$ that is at the same time the lower limit of $\mathscr{A}$ and the upper limit of $\mathscr{U}$. In the pessimistic version of ELECTRE TRI, an alternative $x \in X$ belongs to category $\mathscr{A}$ iff this alternative is declared at least as good as $p$. The central originality of ELECTRE TRI lies in the definition of this "at least as good as" relation that is based on the notions of concordance and non-discordance. Ignoring here the nondiscordance condition, an alternative $x \in X$ is "at least as good as" the profile $p$ if a "sufficient majority" of attributes support this assertion. When preference and indifference thresholds are equal, this is done as follows. A semiorder $T_{i}$ is defined on each attribute. The set of attributes supporting the proposition that $x \in X$ is at least as good as $p$ is simply $\mathcal{T}(x)=\{i \in$ $\left.N: x_{i} T_{i} p_{i}\right\}$. A positive weight $w_{i}$ is assigned to each attribute. These weights are supposed to be normalized so that $\sum_{i=1}^{n} w_{i}=1$. The test for deciding whether the subset of attributes $\mathcal{T}(x)$ is "sufficiently important" is done comparing $\sum_{i \in \mathcal{T}(x)} w_{i}$ to a majority threshold $\lambda \in[0.5,1]$. We have:

$$
x \in \mathscr{A} \Leftrightarrow \sum_{i \in \mathcal{T}(x)} w_{i} \geq \lambda .
$$

Ordered partitions $\langle\mathscr{A}, \mathscr{U}\rangle$ of this type have been studied and characterized in Bouyssou and Marchant (2007a). For the sequel, it will be useful to note that the concordance condition for testing if $x$ is "at least as good as" $p$ only distinguishes two kind of attributes: the ones for which $x_{i} T_{i} p_{i}$ and the ones for which this is not true. It does not make further distinctions among the attributes and, in particular, does not make use of the preference difference between $x_{i}$ and $p_{i}$. Hence, the assignment of an alternative mainly rests on "ordinal considerations".

The model defined below uses similar principles. The main difference with ELECTRE TRI is that the aim of this model is to compare alternatives rather than assigning alternatives to ordered categories. In this model, there is a semiorder $S_{i}$ on each attribute. In order to compare the alternatives 
$x$ and $y$, we first compare each of them to a "reference point" $\pi$ only using "ordinal considerations". Hence, we compute the subsets of attributes $\mathcal{S}(x)=\left\{i \in N: x_{i} S_{i} \pi_{i}\right\}$ and $\mathcal{S}(y)=\left\{i \in N: y_{i} S_{i} \pi_{i}\right\}$. The comparison of $x$ and $y$ is based on the comparison of the subsets $\mathcal{S}(x)$ and $\mathcal{S}(y)$. This comparison uses an "importance relation" that is only required to be monotonic w.r.t. inclusion. The following definition, inspired by Rolland (2003, 2006a,b, 2008,?), formalizes this idea.

Definition 1. A binary relation $\succsim$ is a Relation with a Single Reference Point (or, more briefly, is a RSRP) if:

- for all $i \in N$, there is a semiorder $S_{i}$ on $X_{i}$ (with symmetric part $I_{i}$ and asymmetric part $P_{i}$ ),

- there is an element $\pi \in X$,

- there is a binary relation $\unrhd$ on $2^{N}$ that is monotonic w.r.t. inclusion, i.e., for all $A, B, C, D \subseteq N$,

$$
A \unrhd B \Rightarrow C \unrhd D
$$

whenever, $C \supseteq A, B \supseteq D$, and there are $x, y, z, w \in X$ such that $\mathcal{S}(x)=A, \mathcal{S}(y)=B, \mathcal{S}(z)=C$, and $\mathcal{S}(w)=D$,

such that, for all $x, y \in X$,

$$
x \succsim y \Leftrightarrow \mathcal{S}(x) \unrhd \mathcal{S}(y),
$$

where $\mathcal{S}(x)=\left\{i \in N: x_{i} S_{i} \pi_{i}\right\}$.

The above model uses three parameters: the reference point $\pi$, the semiorders $S_{i}, i=1,2, \ldots, n$, and the importance relation $\unrhd$.

Although this presentation allows to easily grasp the intuition of the model, it is possible to reformulate it using less parameters. Indeed, define, for all $i \in N, \mathcal{A}_{i}=\left\{x_{i} \in X_{i}: x_{i} S_{i} \pi_{i}\right\}$. It is clear that, for all $x \in X$, we have $\mathcal{S}(x)=\left\{i \in N: x_{i} \in \mathcal{A}_{i}\right\}$. Hence, we can alternatively write the above model only using the following parameters: the sets $\mathcal{A}_{i}, i=1,2, \ldots, n$, and the importance relation $\unrhd$.

In this reformulation, alternative $x$ is at least as good as alternative $y$ if the subset of attributes for which $x$ has an evaluation that is "acceptable" (i.e., attributes $i$ such that $x_{i} \in \mathcal{A}_{i}$ ) is "more important" (according to the 
relation $\unrhd$ ) than the subset of attributes for which $y$ has an evaluation that is acceptable.

In Appendix D, we generalize this model to include multiple reference points.

The following lemma shows that a RSRP has a unique representation in terms of the sets $\mathcal{A}_{i}$ and the importance relation $\unrhd$. It makes use of the fact that all attributes have been supposed influential.

Lemma 1. A RSRP has a unique representation in terms of the sets $\mathcal{A}_{i}$, $i \in N$, and the relation $\unrhd$. In this representation we have, for all $i \in N$, $\varnothing \subsetneq \mathcal{A}_{i} \subsetneq X_{i}$.

Proof. Suppose that $\succsim$ is a RSRP having two distinct representations, i.e., that we have

$$
x \succsim y \Leftrightarrow \mathcal{S}(x) \unrhd \mathcal{S}(y) \Leftrightarrow \mathcal{S}^{\prime}(x) \unrhd^{\prime} \mathcal{S}^{\prime}(y),
$$

where $\mathcal{S}(x)=\left\{i \in N: x_{i} \in \mathcal{A}_{i}\right\}$ and $\mathcal{S}^{\prime}(x)=\left\{i \in N: x_{i} \in \mathcal{A}_{i}^{\prime}\right\}$.

Suppose that for some $e_{i} \in X_{i}$, we have $e_{i} \in \mathcal{A}_{i}$ and $e_{i} \notin \mathcal{A}_{i}^{\prime}$. Because attribute $i \in N$ is influential, there are $x_{i}, y_{i}, z_{i}, w_{i} \in X_{i}$ and $a_{-i}, b_{-i} \in X_{-i}$ such that $\left(x_{i}, a_{-i}\right) \succsim\left(y_{i}, b_{-i}\right)$ and $\left(z_{i}, a_{-i}\right) \succsim\left(w_{i}, b_{-i}\right)$.

Because $e_{i} \in \mathcal{A}_{i}$ and $\left(x_{i}, a_{-i}\right) \succsim\left(y_{i}, b_{-i}\right)$, we must have $\left(e_{i}, a_{-i}\right) \succsim\left(y_{i}, b_{-i}\right)$. Similarly, because $e_{i} \notin \mathcal{A}_{i}^{\prime}$ and $\left(z_{i}, a_{-i}\right) \nsucceq\left(w_{i}, b_{-i}\right)$, we must have $\left(e_{i}, a_{-i}\right) \nsucceq$ $\left(w_{i}, b_{-i}\right)$.

Suppose that $y_{i} \in \mathcal{A}_{i}$. Using $\left(e_{i}, a_{-i}\right) \succsim\left(y_{i}, b_{-i}\right)$ and the monotonicity of $\unrhd$, we obtain $\left(e_{i}, a_{-i}\right) \succsim\left(s_{i}, b_{-i}\right)$, for all $s_{i} \in X_{i}$. This is contradictory since we know that $\left(e_{i}, a_{-i}\right) \succsim\left(w_{i}, b_{-i}\right)$.

Suppose that $y_{i} \in \mathcal{A}_{i}^{\prime}$. Using $\left(e_{i}, a_{-i}\right) \succsim\left(y_{i}, b_{-i}\right)$ and the monotonicity of $\unrhd^{\prime}$, we obtain $\left(e_{i}, a_{-i}\right) \succsim\left(s_{i}, b_{-i}\right)$, for all $s_{i} \in X_{i}$. This is contradictory since we know that $\left(e_{i}, a_{-i}\right) \succsim\left(w_{i}, b_{-i}\right)$.

Hence, $y_{i} \notin \mathcal{A}_{i}$ and $y_{i} \notin \mathcal{A}_{i}^{\prime}$. Since we have $\left(e_{i}, a_{-i}\right) \succsim\left(y_{i}, b_{-i}\right)$ and $\left(e_{i}, a_{-i}\right) \nsucceq\left(w_{i}, b_{-i}\right)$, we must have $w_{i} \in \mathcal{A}_{i}$ and $w_{i} \in \mathcal{A}_{i}^{\prime}$.

Because $\left(e_{i}, a_{-i}\right) \succsim\left(y_{i}, b_{-i}\right), y_{i} \notin \mathcal{A}_{i}^{\prime}$ and $e_{i} \notin \mathcal{A}_{i}^{\prime}$, we obtain $\left(e_{i}, a_{-i}\right) \succsim$ $\left(e_{i}, b_{-i}\right)$. Similarly, because $\left(e_{i}, a_{-i}\right) \nsucceq\left(w_{i}, b_{-i}\right), w_{i} \in \mathcal{A}_{i}$ and $e_{i} \in \mathcal{A}_{i}$, we obtain $\left(e_{i}, a_{-i}\right) \nsucceq\left(e_{i}, b_{-i}\right)$, a contradiction.

Hence, we have shown that, for all $i \in N$, we have $\mathcal{A}_{i}=\mathcal{A}_{i}^{\prime}$. It is easy to show that this implies $\unrhd=\unrhd^{\prime}$.

Because attribute $i \in N$ is influential, there are $x_{i}, y_{i}, z_{i}, w_{i} \in X_{i}$ and $a_{-i}, b_{-i} \in X_{-i}$ such that $\left(x_{i}, a_{-i}\right) \succsim\left(y_{i}, b_{-i}\right)$ and $\left(z_{i}, a_{-i}\right) \succsim\left(w_{i}, b_{-i}\right)$. If $\mathcal{A}_{i}=\varnothing$ or $\mathcal{A}_{i}=X_{i}$, we have $\mathcal{S}\left(\left(x_{i}, a_{-i}\right)\right)=\mathcal{S}\left(\left(z_{i}, a_{-i}\right)\right)$ and $\mathcal{S}\left(\left(y_{i}, b_{-i}\right)\right)=$ 
$\mathcal{S}\left(\left(w_{i}, b_{-i}\right)\right)$. Because $\succsim$ is a $\operatorname{RSRP}$, this implies $\left(x_{i}, a_{-i}\right) \succsim\left(y_{i}, b_{-i}\right) \Leftrightarrow$ $\left(z_{i}, a_{-i}\right) \succsim\left(w_{i}, b_{-i}\right)$, a contradiction.

Lemma 2. Let $\succsim$ be $R S R P$ with representation $\mathcal{A}_{i}, i \in N$, and $\unrhd$.

1. $\succsim$ is reflexive iff $\unrhd$ is reflexive,

2. $\succsim$ is complete iff $\unrhd$ is complete,

3. $\succsim$ is transitive iff $\unrhd$ is transitive,

4. $\succsim$ is independent iff, for all $i \in N$ and all $A, B \subseteq N$ such that $i \notin A$ and $i \notin B, A \unrhd B$ iff $A \cup\{i\} \unrhd B \cup\{i\}$.

Proof. We know that $\succsim$ has a unique representation in which, for all $i \in N$, $\varnothing \subsetneq \mathcal{A}_{i} \subsetneq X_{i}$. Hence, for all $A, B \subseteq N$, there are $x, y \in X$ such that $\mathcal{S}(x)=A$ and $\mathcal{S}(y)=B$.

Part 1. Let $x \in X$. Since $\unrhd$ is reflexive, we know that $\mathcal{S}(x) \unrhd \mathcal{S}(x)$, so that $x \succsim x$. Hence, $\succsim$ is reflexive. Conversely, let $A \subseteq N$. We have $\mathcal{S}(x)=A$, for some $x \in X$. Since $x \succsim x$, we obtain $A \unrhd A$. Hence, $\unrhd$ is reflexive.

The proof of Parts 2 and 3 is similar.

Part 4. Suppose that $\succsim$ is not independent so that we have $\left(x_{i}, a_{-i}\right) \succsim$ $\left(x_{i}, b_{-i}\right)$ and $\left(y_{i}, a_{-i}\right) \nsucceq\left(y_{i}, b_{-i}\right)$, for some $i \in N, x_{i}, y_{i} \in X_{i}$, and $a, b \in X$. Since $\succsim$ is a RSRP, it is impossible that $x_{i}, y_{i} \in \mathcal{A}_{i}$ or $x_{i}, y_{i} \notin \mathcal{A}_{i}$. Letting $A=\left\{j \in N \backslash\{i\}: a_{j} \in \mathcal{A}_{j}\right\}$ and $B=\left\{j \in N \backslash\{i\}: b_{j} \in \mathcal{A}_{j}\right\}$, we obtain either $A \unrhd B$ and $A \cup\{i\} \not B \cup\{i\}$ or $A \not B$ and $A \cup\{i\} \unrhd B \cup\{i\}$.

Conversely, suppose that we have $A \unrhd B$ and $A \cup\{i\} \not B \cup\{i\}$. Let $a, b \in X$ be such that $A=\left\{j \in N \backslash\{i\}: a_{j} \in \mathcal{A}_{j}\right\}$ and $B=\{j \in N \backslash\{i\}$ : $\left.b_{j} \in \mathcal{A}_{j}\right\}$. Take $x_{i} \in \mathcal{A}_{i}$ and $y_{i} \notin \mathcal{A}_{i}$. We obtain $\left(x_{i}, a_{-i}\right) \nsucceq\left(x_{i}, b_{-i}\right)$ and $\left(y_{i}, a_{-i}\right) \succsim\left(y_{i}, b_{-i}\right)$. The case $A \not B$ and $A \cup\{i\} \unrhd B \cup\{i\}$ is similar.

A capacity on $N$ is a real valued function $\mu$ on $2^{N}$ such that, for all $A, B \in 2^{N}, A \supseteq B \Rightarrow \mu(A) \geq \mu(B)$. The capacity $\mu$ on $N$ is normalized if, furthermore, $\mu(\varnothing)=0$ and $\mu(N)=1$.

Suppose that $\succsim$ is a RSRP that is a weak order. It follows from the above lemma that $\unrhd$ must be a weak order. Moreover, since $\unrhd$ is monotonic w.r.t. inclusion and $N$ is finite, there is a normalized capacity $\mu$ on $N$ such that $A \unrhd B \Leftrightarrow \mu(A) \geq \mu(B)$. Hence, we have $x \succsim y \Leftrightarrow \mu(\mathcal{S}(x)) \geq \mu(\mathcal{S}(y))$. This shows that a RSRP being a weak order has at most $2^{n}$ distinct equivalence classes. 


\section{Models using traces on levels}

Our central tool for the analysis of RSRP will be the models introduced in Bouyssou and Pirlot (2004b) that induce complete traces on levels on each attribute (see also Greco et al. 2004). We recall here the essential elements of these models.

Definition 2. Let $\succsim$ be a binary relation on a set $X=\prod_{i=1}^{n} X_{i}$. We define the binary relations $\succsim_{i}^{+}, \succsim_{i}^{-}$and $\succsim_{i}^{ \pm}$on $X_{i}$ letting, for all $x_{i}, y_{i} \in X_{i}$,

$$
\begin{aligned}
& x_{i} \succsim_{i}^{+} y_{i} \Leftrightarrow \forall a, b \in X,\left[\left(y_{i}, a_{-i}\right) \succsim^{-} \Rightarrow\left(x_{i}, a_{-i}\right) \succsim b\right], \\
& x_{i} \succsim_{i}^{-} y_{i} \Leftrightarrow \forall a, b \in X,\left[a\left(_{i}, b_{-i}\right) \Rightarrow a \succsim\left(y_{i}, b_{-i}\right)\right], \\
& x_{i} \succsim_{i}^{ \pm} y_{i} \Leftrightarrow\left[x_{i} \succsim_{i}^{+} y_{i} \text { and } x_{i} \succsim_{i}^{-} y_{i}\right] .
\end{aligned}
$$

The relations $\succsim_{i}^{+}, \succsim_{i}^{-}$and $\succsim_{i}^{ \pm}$are traces on the levels of attribute $i \in N$ generated by the relation $\succsim$. It is easy to check that these relations are always reflexive and transitive.

As shown below, the traces on levels combine nicely with the relation $\succsim$.

Lemma 3 (Bouyssou and Pirlot 2004b, Lemma 2). For all $i \in N$ and $x, y, z, w \in X$ :

$$
\begin{gathered}
{\left[x \succsim y, z_{i} \succsim_{i}^{+} x_{i}\right] \Rightarrow\left(z_{i}, x_{-i}\right) \succsim y,} \\
{\left[x \succsim y, y_{i} \succsim_{i}^{-} w_{i}\right] \Rightarrow x \succsim\left(w_{i}, y_{-i}\right),} \\
{\left[z_{i} \succsim_{i}^{ \pm} x_{i}, y_{i} \succsim_{i}^{ \pm} w_{i}\right] \Rightarrow\left\{\begin{array}{l}
x \succsim y \Rightarrow\left(z_{i}, x_{-i}\right) \succsim\left(w_{i}, y_{-i}\right), \\
a n d \\
x \succ y \Rightarrow\left(z_{i}, x_{-i}\right) \succ\left(w_{i}, y_{-i}\right),
\end{array}\right.} \\
{\left[x_{i} \sim_{i}^{ \pm} z_{i}, y_{i} \sim_{i}^{ \pm} w_{i} \text { for all } i \in N\right] \Rightarrow\left\{\begin{array}{l}
x \succsim y \Leftrightarrow z \succsim w, \\
a n d \\
x \succ y \Leftrightarrow z \succ w .
\end{array}\right.}
\end{gathered}
$$

The following conditions will imply the completeness of marginal traces on levels.

Definition 3. We say that $\succsim$ satisfies:

$$
\left.A C 1_{i} \text { if } \begin{array}{c}
\left(x_{i}, a_{-i}\right) \succsim c \\
\text { and } \\
\left(y_{i}, b_{-i}\right) \succsim d
\end{array}\right\} \Rightarrow\left\{\begin{array}{c}
\left(y_{i}, a_{-i}\right) \succsim c \\
\text { or } \\
\left(x_{i}, b_{-i}\right) \succsim d,
\end{array}\right.
$$




$$
\begin{array}{rc}
\left.A C 2_{i} \text { if } \begin{array}{c}
c \succsim\left(y_{i}, a_{-i}\right) \\
\text { and } \\
d \succsim\left(x_{i}, b_{-i}\right)
\end{array}\right\} \Rightarrow\left\{\begin{array}{c}
c \succsim\left(x_{i}, a_{-i}\right) \\
\text { or } \\
d \succsim\left(y_{i}, b_{-i}\right),
\end{array}\right. \\
\left.A C 3_{i} \text { if } \begin{array}{c}
\left(x_{i}, a_{-i}\right) \succsim c \\
\text { and } \\
d \succsim\left(x_{i}, b_{-i}\right)
\end{array}\right\} \Rightarrow\left\{\begin{array}{c}
\left(y_{i}, a_{-i}\right) \succsim c \\
\text { or } \\
d \succsim\left(y_{i}, b_{-i}\right),
\end{array}\right.
\end{array}
$$

for all $a, b, c, d \in X$ and all $x_{i}, y_{i} \in X_{i}$. We say that $\succsim$ satisfies $A C 1$ (resp. $A C 2, A C 3)$ if it satisfies $A C 1_{i}$ (resp. $\left.A C 2_{i}, A C 3_{i}\right)$ for all $i \in N$.

Lemma 4 (Bouyssou and Pirlot 2004b, Lemma 3). We have:

1. $A C 1_{i} \Leftrightarrow \succsim_{i}^{+}$is complete $\Leftrightarrow\left[\operatorname{Not}\left[y_{i} \succsim_{i}^{+} x_{i}\right] \Rightarrow x_{i} \succsim_{i}^{+} y_{i}\right]$.

2. $A C 2_{i} \Leftrightarrow \succsim_{i}^{-}$is complete $\Leftrightarrow\left[\operatorname{Not}\left[y_{i} \succsim_{i}^{-} x_{i}\right] \Rightarrow x_{i} \succsim_{i}^{-} y_{i}\right]$.

3. $A C 3_{i} \Leftrightarrow\left[\operatorname{Not}\left[y_{i} \succsim_{i}^{+} x_{i}\right] \Rightarrow x_{i} \succsim_{i}^{-} y_{i}\right] \Leftrightarrow\left[N o t\left[x_{i} \succsim_{i}^{-} y_{i}\right] \Rightarrow y_{i} \succsim_{i}^{+} x_{i}\right]$.

4. $\left[A C 1_{i}, A C 2_{i}\right.$ and $\left.A C 3_{i}\right] \Leftrightarrow \succsim_{i}^{ \pm}$is complete.

5. In the class of all semiorders on $X, A C 1, A C 2$ and $A C 3$ are independent conditions.

The following shows the consequences of having complete traces on each attribute in terms of numerical representation.

Proposition 1 (Bouyssou and Pirlot 2004b, Theorem 2). Let $\succsim$ be a binary relation on a set $X=\prod_{i=1}^{n} X_{i}$. Suppose that, for all $i \in N$, the set $X_{i} / \sim_{i}^{ \pm}$ is at most countably infinite. Then there are real-valued functions $u_{i}$ on $X_{i}$ and a real-valued function $F$ on $\left[\prod_{i=1}^{n} u_{i}\left(X_{i}\right)\right]^{2}$ such that, for all $x, y \in X$ :

$$
x \succsim y \Leftrightarrow F\left(\left[u_{i}\left(x_{i}\right)\right] ;\left[u_{i}\left(y_{i}\right)\right]\right) \geq 0,
$$

with $F$ is increasing in its first $n$ arguments and decreasing in its last $n$ arguments iff $\succsim$ satisfies $A C 1, A C 2$ and $A C 3$.

We refer to Bouyssou and Pirlot (2004b) for the analysis of model (M) in the general case, i.e., when the set $X_{i} / \sim_{i}^{ \pm}$can be uncountable (this requires conditions guaranteeing that the relations $\succsim_{i}^{ \pm}$have a numerical representation). This will not be useful here. Indeed, a characteristic feature of the models studied here is that they generate complete traces $\succsim_{i}^{ \pm}$that are "rough", only distinguishing a very limited number of distinct equivalence classes.

Let us conclude with a brief analysis of the very particular situation in which $\succsim$ is a weak order. 
Definition 4. Let $\succsim$ be a weak order on $X$. Attribute $i \in N$ is said to be weakly separable if $\left(x_{i}, a_{-i}\right) \succ\left(y_{i}, a_{-i}\right)$ for some $x_{i}, y_{i} \in X_{i}$ and some $a_{-i} \in X_{-i}$ implies $\left(x_{i}, b_{-i}\right) \succsim\left(y_{i}, b_{-i}\right)$, for all $b_{-i} \in X_{-i}$. The relation $\succsim$ is said to be weakly separable if all attributes $i \in N$ are weakly separable.

The following lemma show that for weak orders, the three conditions $A C 1_{i}, A C 2_{i}$, and $A C 3_{i}$ are equivalent. Furthermore, they are equivalent to requiring that attribute $i \in N$ is weakly separable.

Lemma 5 (Bouyssou and Pirlot 2004b, Lemma 5). Let $\succsim$ be a weak order on $X$. Conditions $A C 1_{i}, A C 2_{i}$ and $A C 3_{i}$ are equivalent. They hold iff attribute $i \in N$ is weakly separable.

When $\succsim$ is a weakly separable weak order, i.e., a weak order satisfying $A C 1, A C 2$ and $A C 3$, it is possible to further specify the numerical representation given by model $(\mathrm{M})$. We have:

Proposition 2 (Bouyssou and Pirlot 2004b, Proposition 8). Let $\succsim$ be a weak order on a set $X=\prod_{i=1}^{n} X_{i}$ such that the set $X / \sim$ is at most countably infinite. Then there are real-valued functions $u_{i}$ on $X_{i}$ and a real-valued function $U$ on $\prod_{i=1}^{n} u_{i}\left(X_{i}\right)$ such that, for all $x, y \in X$ :

$$
x \succsim y \Leftrightarrow U\left(\left[u_{i}\left(x_{i}\right)\right]\right) \geq U\left(\left[u_{i}\left(y_{i}\right)\right]\right),
$$

with $U$ is nondecreasing in each of its arguments decreasing in its last $n$ arguments iff $\succsim$ is weakly separable.

\section{Results}

\subsection{A characterization of $R S R P$}

Our analysis of RSRP is based on the following two lemmas.

Lemma 6. If $\succsim$ is a $R S R P$, then, for all $i \in N$, the relation $\succsim_{i}^{ \pm}$is a weak order having two distinct equivalence classes.

Proof. Let $\succsim$ be a RSRP. Lemma 1 has shown that it has a unique representation using the sets $\mathcal{A}_{i}$ and the relation $\unrhd$. Using the definition of a RSRP, it is easy to see that if $x_{i}, y_{i} \in \mathcal{A}_{i}$ or if $x_{i}, y_{i} \notin \mathcal{A}_{i}$, we have $x_{i} \sim_{i}^{ \pm} y_{i}$. Moreover, if $x_{i} \in \mathcal{A}_{i}$ and $y_{i} \notin \mathcal{A}_{i}$, the monotonicity of $\unrhd$ w.r.t. inclusion implies $x_{i} \succsim_{i}^{ \pm} y_{i}$. Hence, the relation $\succsim_{i}^{ \pm}$is a weak order having at most two distinct equivalence classes. 
Because we have supposed that each $i \in N$ is influential, there are $x_{i}, y_{i}, z_{i}, w_{i} \in X_{i}$ and $a, b \in X$ such that $\left(x_{i}, a_{-i}\right) \succsim\left(y_{i}, b_{-i}\right)$ and $\left(z_{i}, a_{-i}\right) \nsucceq$ $\left(w_{i}, b_{-i}\right)$. If $z_{i} \succsim_{i}^{+} x_{i}$ and $y_{i} \succsim_{i}^{-} w_{i},\left(x_{i}, a_{-i}\right) \succsim\left(y_{i}, b_{-i}\right)$ implies $\left(z_{i}, a_{-i}\right) \succsim$ $\left(w_{i}, b_{-i}\right)$, a contradiction. Hence we must have either $x_{i} \succ_{i}^{+} z_{i}$ or $w_{i} \succ_{i}^{-} y_{i}$. Either case implies that the weak order $\succsim_{i}^{ \pm}$has at least two distinct equivalence classes.

Remark 1. Lemmas 4 and 6 imply that a RSRP satisfies $A C 1, A C 2$ and $A C 3$. Furthermore all sets $X_{i} / \sim_{i}^{ \pm}$are finite, having two distinct elements. Hence, if a RSRP also has a representation in (M). It is not difficult to figure out the particular form of model $(\mathrm{M})$ that leads to a RSRP. Indeed, it suffices to consider, on each $i \in N$, a function $u_{i}$ such that, for all $x_{i} \in X_{i}$,

$$
u_{i}\left(x_{i}\right)= \begin{cases}1 & \text { if } x_{i} \in \mathcal{A}_{i} \\ 0 & \text { if } x_{i} \notin \mathcal{A}_{i}\end{cases}
$$

With the above definition, we clearly have $u_{i}\left(x_{i}\right) \geq u_{i}\left(y_{i}\right) \Leftrightarrow x_{i} \succsim_{i}^{ \pm} y_{i}$.

We define the function $F$ letting:

$$
F\left(\left[u_{i}\left(x_{i}\right)\right] ;\left[u_{i}\left(y_{i}\right)\right]\right)= \begin{cases}+\exp \left(\sum_{i=1}^{n}\left(u_{i}\left(x_{i}\right)-u_{i}\left(y_{i}\right)\right)\right) & \text { if } x \succsim y, \\ -\exp \left(\sum_{i=1}^{n}\left(u_{i}\left(y_{i}\right)-u_{i}\left(x_{i}\right)\right)\right) & \text { otherwise. }\end{cases}
$$

Using the definition of the functions $u_{i}$ and the fact that $\unrhd$ is monotonic w.r.t. inclusion, it is easy to show, using (4), that $F$ is well defined and increasing (resp. decreasing) in its first (resp. last) $n$ arguments.

Lemma 7. If $\succsim$ is a relation on $X$ such that, for all $i \in N$, the relation $\succsim_{i}^{ \pm}$ is a weak order having two distinct equivalence classes, then $\succsim$ is a RSRP.

Proof. For all $i \in N$, define $\mathcal{A}_{i}$ as the set of elements of $X_{i}$ in the first equivalence class of $\succsim_{i}^{ \pm}$. Define the relation $\unrhd$ letting, for all $A, B \subseteq N$, $A \unrhd B$ if $x \succsim y$, for some $x, y \in X$ such that $\mathcal{S}(x)=A$ and $\mathcal{S}(y)=B$. We have to show that, for all $x, y \in X, x \succsim y \Leftrightarrow \mathcal{S}(x) \unrhd \mathcal{S}(y)$ and that $\unrhd$ is monotonic w.r.t. inclusion. If $x \succsim y$, the definition of $\unrhd$ implies that $\mathcal{S}(x) \unrhd \mathcal{S}(y)$. Suppose now that $\mathcal{S}(x) \unrhd \mathcal{S}(y)$. By construction, this implies that, for some $z, w \in X$ we have $z \succsim w, \mathcal{S}(z)=\mathcal{S}(x)$ and $\mathcal{S}(w)=\mathcal{S}(y)$. This implies that, for all $i \in N, x_{i} \sim_{i}^{ \pm} z_{i}$ and $y_{i} \sim_{i}^{ \pm} w_{i}$. Using (4), we obtain $x \succsim y$.

It remains to prove that $\unrhd$ is monotonic w.r.t. inclusion. 
Suppose that for some $x, y, z, w \in X$ we have $\mathcal{S}(z) \supseteq \mathcal{S}(x), \mathcal{S}(y) \supseteq$ $\mathcal{S}(w)$, and $\mathcal{S}(x) \unrhd \mathcal{S}(w)$. By construction of the sets $\mathcal{A}_{i}, \mathcal{S}(z) \supseteq \mathcal{S}(x)$ and $\mathcal{S}(y) \supseteq \mathcal{S}(w)$ imply that, for all $i \in N$, we have $z_{i} \succsim_{i}^{ \pm} x_{i}$ and $y_{i} \succsim_{i}^{ \pm} w_{i}$. Since $\mathcal{S}(x) \unrhd \mathcal{S}(y)$, we have $x \succsim y$. Using (3), we obtain $z \succsim w$, so that $\mathcal{S}(z) \unrhd \mathcal{S}(w)$.

In view of the above two lemmas, a characterization of RSRP will be at hand if we impose conditions guaranteeing that all relations $\succsim_{i}^{ \pm}$are weak orders having two distinct equivalence classes.

Definition 5 (Conditions $\left.A C 1^{*}, A C 2^{*}, A C 3^{*}, A C 4^{*}\right)$. We say that $\succsim$ satisfies:

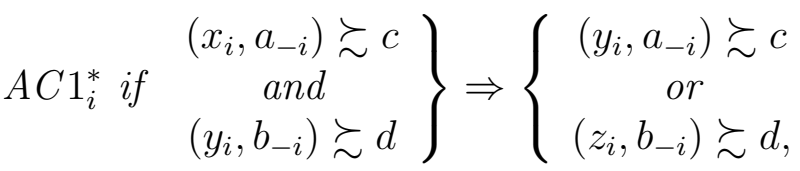

$$
\begin{aligned}
& \left.A C 2_{i}^{*} \text { if } \begin{array}{c}
c \succsim\left(y_{i}, a_{-i}\right) \\
\text { and } \\
d \succsim\left(z_{i}, b_{-i}\right)
\end{array}\right\} \Rightarrow\left\{\begin{array}{c}
c \succsim\left(x_{i}, a_{-i}\right) \\
\text { or } \\
d \succsim\left(y_{i}, b_{-i}\right),
\end{array}\right. \\
& \left.A C 3_{i}^{*} \text { if } \begin{array}{c}
\left(x_{i}, a_{-i}\right) \succsim c \\
\text { and } \\
d \succsim\left(z_{i}, b_{-i}\right)
\end{array}\right\} \Rightarrow\left\{\begin{array}{c}
\left(y_{i}, a_{-i}\right) \succsim c \\
\text { or } \\
d \succsim\left(y_{i}, b_{-i}\right),
\end{array}\right. \\
& \left.A C 4_{i}^{*} \text { if } \begin{array}{c}
c \succsim\left(y_{i}, a_{-i}\right) \\
\text { and } \\
\left(y_{i}, b_{-i}\right) \succsim d
\end{array}\right\} \Rightarrow\left\{\begin{array}{c}
c \succsim\left(x_{i}, a_{-i}\right) \\
\text { or } \\
\left(z_{i}, b_{-i}\right) \succsim d,
\end{array}\right.
\end{aligned}
$$

for all $a, b, c, d \in X$ and all $x_{i}, y_{i}, z_{i} \in X_{i}$. We say that $\succsim$ satisfies $A C 1^{*}$ (resp. $A C 2^{*}, A C 3^{*}, A C 4^{*}$ ) if it satisfies $A C 1_{i}^{*}$ (resp. $A C 2_{i}^{*}, A C 3_{i}^{*}, A C 4_{i}^{*}$ ) for all $i \in N$.

Let us show that the above four conditions hold for a RSRP.

Lemma 8. If $\succsim$ is a $R S R P$ then it satisfies $A C 1^{*}, A C 2^{*}, A C 3^{*}$, and $A C 4^{*}$.

Proof. Suppose that $A C 1_{i}^{*}$ is violated, so that we have $\left(x_{i}, a_{-i}\right) \succsim c,\left(y_{i}, b_{-i}\right) \succsim$ $d,\left(y_{i}, a_{-i}\right) \nsucceq c$, and $\left(z_{i}, b_{-i}\right) \nsucceq d$, for some $a, b, c, d \in X$ and some $x_{i}, y_{i}, z_{i} \in$ $X_{i}$. Since $\left(x_{i}, a_{-i}\right) \succsim c$ and $\left(y_{i}, a_{-i}\right) \succsim c$, we have $N o t\left[y_{i} \succsim_{i}^{+} x_{i}\right]$. In a RSRP, this can only happen if $y_{i} \notin \mathcal{A}_{i}$. Since $y_{i} \notin \mathcal{A}_{i}$, we have $\mathcal{S}\left(\left(z_{i}, a_{-i}\right)\right) \supseteq$ $\mathcal{S}\left(\left(y_{i}, a_{-i}\right)\right)$. Because $\left(y_{i}, b_{-i}\right) \succsim d$, we know that $\mathcal{S}\left(\left(y_{i}, b_{-i}\right)\right) \unrhd \mathcal{S}(d)$. Using the monotonicity of $\unrhd$ w.r.t. inclusion, we obtain $\mathcal{S}\left(\left(z_{i}, b_{-i}\right)\right) \unrhd \mathcal{S}(d)$, so that $\left(z_{i}, b_{-i}\right) \succsim d$, a contradiction. The proof of the other parts is similar. 
The consequences of the above four conditions on the relations $\succsim_{i}^{+}$and $\succsim_{i}^{-}$are detailed below.

Lemma 9. For all $x_{i}, y_{i}, z_{i} \in X_{i}$,

1. $A C 1_{i}^{*} \Leftrightarrow\left[\operatorname{Not}\left[y_{i} \succsim_{i}^{+} x_{i}\right] \Rightarrow z_{i} \succsim_{i}^{+} y_{i}\right]$,

2. $A C 2_{i}^{*} \Leftrightarrow\left[\operatorname{Not}\left[y_{i} \succsim_{i}^{-} x_{i}\right] \Rightarrow z_{i} \succsim_{i}^{-} y_{i}\right]$,

3. $A C 3_{i}^{*} \Leftrightarrow\left[\operatorname{Not}\left[y_{i} \succsim_{i}^{+} x_{i}\right] \Rightarrow z_{i} \succsim_{i}^{-} y_{i}\right]$,

4. $A C 4_{i}^{*} \Leftrightarrow\left[\operatorname{Not}\left[y_{i} \succsim_{i}^{-} x_{i}\right] \Rightarrow z_{i} \succsim_{i}^{+} y_{i}\right]$.

Proof. We prove Part 1, the proof of the other parts being similar. Negating $A C 1_{i}^{*}$ is equivalent to supposing that $\left(x_{i}, a_{-i}\right) \succsim c,\left(y_{i}, b_{-i}\right) \succsim d,\left(y_{i}, a_{-i}\right) \succsim c$, $\left(z_{i}, b_{-i}\right) \nsucceq d$, for some $a, b, c, d \in X$ and some $x_{i}, y_{i}, z_{i} \in X_{i}$. This is equivalent to saying that we have $\operatorname{Not}\left[y_{i} \succsim_{i}^{+} x_{i}\right]$ and $\operatorname{Not}\left[z_{i} \succsim_{i}^{+} y_{i}\right]$.

Combining Lemmas 4 and 9 proves the following.

Lemma 10. 1. $A C 1_{i}^{*} \Rightarrow A C 1_{i}$,

2. $A C 2_{i}^{*} \Rightarrow A C 2_{i}$,

3. $A C 3_{i}^{*} \Rightarrow A C 3_{i}$,

4. $A C 4_{i}^{*} \Rightarrow A C 3_{i}$.

A crucial consequence of the combination of $A C 1^{*}, A C 2^{*}, A C 3^{*}$ and $A C 4^{*}$ is presented below.

Lemma 11. The relation $\succsim$ satisfies $A C 1_{i}^{*}, A C 2_{i}^{*}, A C 3_{i}^{*}$, and $A C 4_{i}^{*}$ iff the binary relation $\succsim_{i}^{ \pm}$is a weak order having two distinct equivalence classes.

Proof. $\left[A C 1_{i}^{*}, A C 2_{i}^{*}, A C 3_{i}^{*}, A C 4_{i}^{*}\right] \Rightarrow\left[\succsim_{i}^{ \pm}\right.$is a weak order having at most two distinct equivalence classes]. Using Lemma 10, we know that $A C 1_{i}$, $A C 2_{i}$ and $A C 3_{i}$ hold, so that, using Lemma $4, \succsim_{i}^{ \pm}$is a weak order. Suppose that we have, for some $x_{i}, y_{i}, z_{i} \in X_{i}, x_{i} \succ_{i}^{ \pm} y_{i}$ and $y_{i} \succ_{i}^{ \pm} z_{i}$. By construction, $x_{i} \succ_{i}^{ \pm} y_{i}$ implies either

$$
\begin{aligned}
& {\left[x_{i} \succ_{i}^{+} y_{i} \text { and } x_{i} \succsim_{i}^{-} y_{i}\right] \text { or }} \\
& {\left[x_{i} \succsim_{i}^{+} y_{i} \text { and } x_{i} \succ_{i}^{-} y_{i}\right] .}
\end{aligned}
$$

Similarly, $y_{i} \succ_{i}^{ \pm} z_{i}$ implies either

$$
\begin{aligned}
& {\left[y_{i} \succ_{i}^{+} z_{i} \text { and } y_{i} \succsim_{i}^{-} z_{i}\right] \text { or }} \\
& {\left[y_{i} \succsim_{i}^{+} z_{i} \text { and } y_{i} \succ_{i}^{-} z_{i}\right] .}
\end{aligned}
$$


The combination of (5a) and (5c) violates Lemma 9.1 (i.e., Part 1 of Lemma 9) since it implies $\operatorname{Not}\left[y_{i} \succsim_{i}^{+} x_{i}\right]$ and $\operatorname{Not}\left[z_{i} \succsim_{i}^{+} y_{i}\right]$. The combination of $(5 \mathrm{~b})$ and (5d) violates Lemma 9.2 since it implies $\operatorname{Not}\left[y_{i} \succsim_{i}^{-} x_{i}\right]$ and $\operatorname{Not}\left[z_{i} \succsim_{i}^{-}\right.$ $\left.y_{i}\right]$. The combination of $(5 \mathrm{a})$ and $(5 \mathrm{~d})$ violates Lemma 9.3 since it implies $\operatorname{Not}\left[y_{i} \succsim_{i}^{+} x_{i}\right]$ and $\operatorname{Not}\left[z_{i} \succsim_{i}^{-} y_{i}\right]$. Finally, the combination of $(5 \mathrm{~b})$ and $(5 \mathrm{c})$ violates Lemma 9.4 since it implies $\operatorname{Not}\left[y_{i} \succsim_{i}^{-} x_{i}\right]$ and $\operatorname{Not}\left[z_{i} \succsim_{i}^{+} y_{i}\right]$.

$\left[\succsim_{i}^{ \pm}\right.$is a weak order having at most two distinct equivalence classes $] \Rightarrow$ $\left[A C 1_{i}^{*}, A C 2_{i}^{*}, A C 3_{i}^{*}, A C 4_{i}^{*}\right]$. Suppose that $\left(x_{i}, a_{-i}\right) \succsim c$, and $\left(y_{i}, b_{-i}\right) \succsim d$. If $\left(y_{i}, a_{-i}\right) \nsucceq c$, we know that $x_{i} \succ_{i}^{+} y_{i}$, so that $x_{i} \succ_{i}^{ \pm} y_{i}$. Since $\succsim_{i}^{ \pm}$is a weak order having only two distinct equivalence classes, this implies that, for all $z_{i} \in X_{i}, z_{i} \succsim_{i}^{ \pm} y_{i}$, so that $z_{i} \succsim_{i}^{+} y_{i}$. Hence, $\left(y_{i}, b_{-i}\right) \succsim d \operatorname{implies}\left(z_{i}, b_{-i}\right) \succsim d$. This shows that $A C 1_{i}^{*}$ holds. The proof for the other three conditions is similar.

The proof is completed observing that, since all attributes are influential, all relations $\succsim_{i}^{ \pm}$have at least two equivalence classes.

Our first characterization of RSRP is as follows.

Theorem 1. Let $\succsim$ be a binary relation on a set $X=\prod_{i=1}^{n} X_{i}$. The relation $\succsim$ is a Relation with a Single Reference Point iff it satisfies $A C 1^{*}, A C 2^{*}$, $A C 3^{*}$, and $A C 4^{*}$.

Proof. Necessity results from Lemma 8. Sufficiency results from Lemmas 7 and 11.

The conditions used in the above theorem are independent, even when attention is restricted to a class of well behaved relations on $X$.

Lemma 12. In the class of all semiorders on $X$, conditions $A C 1^{*}, A C 2^{*}$, $A C 3^{*}$, and $A C 4^{*}$ are independent.

Proof. See Appendix A.

\subsection{An alternative characterization of $R S R P$}

We have seen above a RSRP is characterized by the conjunction of conditions $A C 1_{i}^{*}, A C 2_{i}^{*}, A C 3_{i}^{*}$ and $A C 4_{i}^{*}$. A drawback of this result is that it does not explicitly use conditions $A C 1_{i}, A C 2_{i}$ and $A C 3_{i}$ that characterize model (M). We show here how to weaken conditions $A C 1_{i}^{*}, A C 2_{i}^{*}, A C 3_{i}^{*}$, $A C 4_{i}^{*}$ so as to make them independent from conditions $A C 1_{i}, A C 2_{i}$ and $A C 3_{i}$. This will allow us to exactly state what must be added to the conditions characterizing model (M) to obtain RSRP. 
Definition 6 (Conditions $A C 1^{* *}, A C 2^{* *}, A C 3^{* *}, A C 4^{* *}$ ). We say that $\succsim$ satisfies:

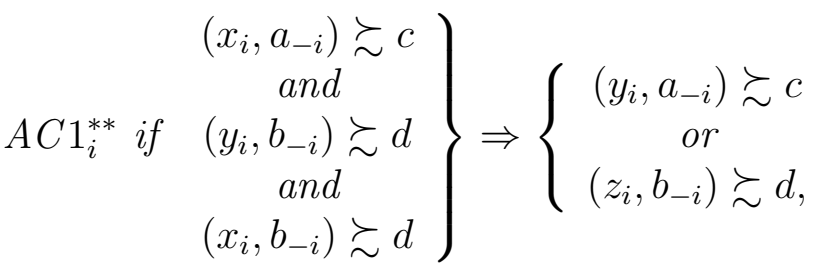

$$
\begin{aligned}
& \left.\begin{array}{rl}
c & \succsim\left(y_{i}, a_{-i}\right) \\
A C 2_{i}^{* *} \text { if } \quad & d \succsim\left(z_{i}, b_{-i}\right) \\
& \text { and } \\
c & \succsim\left(z_{i}, a_{-i}\right)
\end{array}\right\} \Rightarrow\left\{\begin{array}{c}
c \succsim\left(x_{i}, a_{-i}\right) \\
\text { or } \\
d \succsim\left(y_{i}, b_{-i}\right),
\end{array}\right. \\
& \left.A C 3_{i}^{* *} \text { if } \begin{array}{c}
\left(x_{i}, a_{-i}\right) \succsim c \\
\text { and } \\
d \succsim\left(z_{i}, b_{-i}\right)
\end{array}\right\} \Rightarrow\left\{\begin{array}{c}
\left(y_{i}, a_{-i}\right) \succsim c \\
\text { or } \\
d \succsim\left(y_{i}, b_{-i}\right) \\
\text { or } \\
d \succsim\left(x_{i}, b_{-i}\right),
\end{array}\right. \\
& \left.\begin{array}{c}
c \succsim\left(y_{i}, a_{-i}\right) \\
\text { and } \\
A C 4_{i}^{* *} \text { if } \quad\left(y_{i}, b_{-i}\right) \succsim d \\
\text { and } \\
\left(x_{i}, b_{-i}\right) \succsim d
\end{array}\right\} \Rightarrow\left\{\begin{array}{c}
c \succsim\left(x_{i}, a_{-i}\right) \\
o r \\
\left(z_{i}, b_{-i}\right) \succsim d,
\end{array}\right.
\end{aligned}
$$

for all $a, b, c, d \in X$ and all $x_{i}, y_{i}, z_{i} \in X_{i}$. We say that $\succsim$ satisfies $A C 1^{* *}$ (resp. $A C 2^{* *}, A C 3^{* *}, A C 4^{* *}$ ) if it satisfies $A C 1_{i}^{* *}$ (resp. $A C 2_{i}^{* *}, A C 3_{i}^{* *}$, $\left.A C 4_{i}^{* *}\right)$ for all $i \in N$.

Lemma 13. Let $\succsim$ be a binary relation on $X$. We have:

1. $\succsim$ satisfies $A C 1_{i}^{*}$ iff it satisfies $A C 1_{i}$ and $A C 1_{i}^{* *}$,

2. $\succsim$ satisfies $A C 2_{i}^{*}$ iff it satisfies $A C 2_{i}$ and $A C 2_{i}^{* *}$,

3. $\succsim$ satisfies $A C 3_{i}^{*}$ iff it satisfies $A C 3_{i}$ and $A C 3_{i}^{* *}$,

4. $\succsim$ satisfies $A C 4_{i}^{*}$ iff it satisfies $A C 3_{i}$ and $A C 4_{i}^{* *}$.

Proof. Part 1. It is clear that $A C 1_{i}^{*}$ implies $A C 1_{i}^{* *}$ (since the latter condition amount to add a premise to the former condition) and $A C 1_{i}$ (in view of Lemma 10). Let us show that the reverse implication holds. Suppose that $A C 1_{i}^{*}$ is violated, so that we have: $\left(x_{i}, a_{-i}\right) \succsim c,\left(y_{i}, b_{-i}\right) \succsim d,\left(y_{i}, a_{-i}\right) \succsim c$, 
$\left(z_{i}, b_{-i}\right) \nsucceq d$, for some $a, b, c, d \in X$ and some $x_{i}, y_{i}, z_{i} \in X_{i}$. Since $A C 1_{i}$ holds, we know that $\succsim_{i}^{+}$is a weak order. In view of the fact that $\left(x_{i}, a_{-i}\right) \succsim c$ and $\left(y_{i}, a_{-i}\right) \nsucceq c$, we must have $x_{i} \succsim_{i}^{+} y_{i}$. Since $\left(y_{i}, b_{-i}\right) \succsim d$, we obtain $\left(x_{i}, b_{-i}\right) \succsim d$. Hence we have: $\left(x_{i}, a_{-i}\right) \succsim c,\left(y_{i}, b_{-i}\right) \succsim d,\left(x_{i}, b_{-i}\right) \succsim d$, $\left(y_{i}, a_{-i}\right) \nsucceq c,\left(z_{i}, b_{-i}\right) \nsucceq d$, violating $A C 1_{i}^{* *}$.

Part 2. It is clear that $A C 2_{i}^{*}$ implies $A C 2_{i}^{* *}$ (since the latter condition amount to add a premise to the former condition) and $A C 2_{i}$ (in view of Lemma 10). Let us show that the reverse implication holds. Suppose that $A C 2_{i}^{*}$ is violated, so that we have: $c \succsim\left(y_{i}, a_{-i}\right), d \succsim\left(z_{i}, b_{-i}\right), c \succsim\left(x_{i}, a_{-i}\right)$, $d \nsucceq\left(y_{i}, b_{-i}\right)$, for some $a, b, c, d \in X$ and some $x_{i}, y_{i}, z_{i} \in X_{i}$. Since $A C 2_{i}$ holds, we know that $\succsim_{i}^{-}$is a weak order. In view of the fact that $d \succsim\left(z_{i}, b_{-i}\right)$ and $d \nsucceq\left(y_{i}, b_{-i}\right)$, we must have $y_{i} \succsim_{i}^{-} z_{i}$. Since $c \succsim\left(y_{i}, a_{-i}\right)$, we obtain $c \succsim\left(z_{i}, a_{-i}\right)$. Hence we have: $c \succsim\left(y_{i}, a_{-i}\right), d \succsim\left(z_{i}, b_{-i}\right), c \succsim\left(z_{i}, a_{-i}\right)$, $c \nsucceq\left(x_{i}, a_{-i}\right), d \nsucceq\left(y_{i}, b_{-i}\right)$, violating $A C 2_{i}^{* *}$.

Part 3. It is clear that $A C 3_{i}^{*}$ implies $A C 3_{i}^{* *}$ (since the latter condition amount to add a possible conclusion to the former condition) and $A C 3_{i}$ (in view of Lemma 10). Let us show that the reverse implication holds. Suppose that $A C 3_{i}^{*}$ is violated, so that we have: $\left(x_{i}, a_{-i}\right) \succsim c, d \succsim\left(z_{i}, b_{-i}\right)$, $\left(y_{i}, a_{-i}\right) \nsucceq c, d \nsucceq\left(y_{i}, b_{-i}\right)$, for some $a, b, c, d \in X$ and some $x_{i}, y_{i}, z_{i} \in X_{i}$. Since $\left(x_{i}, a_{-i}\right) \succsim c$ and $\left(y_{i}, a_{-i}\right) \nsucceq c$, it is impossible that $y_{i} \succsim_{i}^{+} x_{i}$. Using $A C 3_{i}$ and Lemma 4 implies that we must have $x_{i} \succsim_{i}^{-} y_{i}$. Hence, because $d \nsucceq\left(y_{i}, b_{-i}\right)$, we must have $d \nsucceq\left(x_{i}, b_{-i}\right)$. Hence we have: $\left(x_{i}, a_{-i}\right) \succsim c$, $d \succsim\left(z_{i}, b_{-i}\right),\left(y_{i}, a_{-i}\right) \succsim c, d \succsim\left(y_{i}, b_{-i}\right), d \succsim\left(x_{i}, b_{-i}\right)$, violating $A C 3_{i}^{* *}$.

Part 4. It is clear that $A C 4_{i}^{*}$ implies $A C 4_{i}^{* *}$ (since the latter condition amount to add a premise to the former condition) and $A C 3_{i}$ (in view of Lemma 10). Let us show that the reverse implication holds. Suppose that $A C 4_{i}^{*}$ is violated, so that we have: $c \succsim\left(y_{i}, a_{-i}\right),\left(y_{i}, b_{-i}\right) \succsim d, c \succsim\left(x_{i}, a_{-i}\right)$, $\left(z_{i}, b_{-i}\right) \nsucceq d$, for some $a, b, c, d \in X$ and some $x_{i}, y_{i}, z_{i} \in X_{i}$. Since $c \succsim$ $\left(y_{i}, a_{-i}\right)$ and $c \succsim\left(x_{i}, a_{-i}\right)$, it is impossible that $y_{i} \succsim_{i}^{-} x_{i}$. Using $A C 3_{i}$ and Lemma 4 implies that we must have $x_{i} \succsim_{i}^{+} y_{i}$. Since $\left(y_{i}, b_{-i}\right) \succsim d$, we therefore obtain $\left(x_{i}, b_{-i}\right) \succsim d$. Hence we have: $c \succsim\left(y_{i}, a_{-i}\right),\left(y_{i}, b_{-i}\right) \succsim d,\left(x_{i}, b_{-i}\right) \succsim d$, $c \succsim\left(x_{i}, a_{-i}\right),\left(z_{i}, b_{-i}\right) \nsucceq d$, violating $A C 4_{i}^{* *}$.

Combining the above lemma with Theorem 1 proves the following:

Theorem 2. Let $\succsim$ be a binary relation on a set $X=\prod_{i=1}^{n} X_{i}$. The relation $\succsim$ is a Relation with a Single Reference Point iff it satisfies $A C 1, A C 2, A C 3$, $A C 1^{* *}, A C 2^{* *}, A C 3^{* *}$, and $A C 4^{* *}$. 
The following lemma shows that the conditions used in the above result are independent even when attention is restricted to a class of well behaved relations on $X$.

Lemma 14. In the class of all semiorders on $X$, conditions $A C 1_{i}, A C 2_{i}$, $A C 3_{i}, A C 1^{* *}, A C 2^{* *}, A C 3^{* *}$, and $A C 4^{* *}$ are independent.

Proof. See Appendix B.

\section{Relation to concordance relations}

In view of Lemma 2.4, it is easy to build examples showing that a RSRP does not have to be independent. It is well known that concordance relations are independent (Bouyssou and Pirlot 2005b, 2007). Using this observation, Rolland (2003, 2006a, 2008) has concluded that models using reference points were more "flexible" than concordance relations. This section is devoted to a precise analysis of the links between concordance relations and RSRP.

We first recall the definition of a concordance relation (Bouyssou and Pirlot 2005b, 2007) when $\succsim$ is reflexive (since a concordance relation is independent, it is easy to check that it is either reflexive or irreflexive).

Definition 7. Let $\succsim$ be a reflexive binary relation on $X$. We say that $\succsim$ is a concordance relation with attribute transitivity (or, more briefly, that $\succsim$ is a $C R$-AT) if there are:

- $a$ semiorder $T_{i}$ on each $X_{i}(i=1,2, \ldots, n)$,

- a binary relation $\unrhd_{C}$ between subsets of $N$ having $N$ for union that is monotonic w.r.t. inclusion, i.e., for all $A, B, C, D \subseteq N$ such that $A \cup B=N$ and $C \cup D=N$,

$$
\left[A \unrhd_{C} B, C \supseteq A, B \supseteq D\right] \Rightarrow C \unrhd_{C} D,
$$

such that, for all $x, y \in X$,

$$
x \succsim y \Leftrightarrow T(x, y) \unrhd_{C} T(y, x)
$$

where $T(x, y)=\left\{i \in N: x_{i} T_{i} y_{i}\right\}$. 
Hence, when $\succsim$ is a CR-AT, the preference between $x$ and $y$ only depends on the subsets of attributes favoring $x$ or $y$ in terms of the semiorder $T_{i}$. It does not depend on preference differences between the various levels on each attribute besides the distinction between levels indicated by $T_{i}$.

Definition 8. Let $\succsim$ be a binary relation on a set $X=\prod_{i=1}^{n} X_{i}$. We define the binary relations $\succsim_{i}^{*}$ and $\succsim_{i}^{* *}$ on $X_{i}^{2}$ letting, for all $x_{i}, y_{i}, z_{i}, w_{i} \in X_{i}$,

$$
\begin{aligned}
&\left(x_{i}, y_{i}\right) \succsim_{i}^{*}\left(z_{i}, w_{i}\right) \Leftrightarrow \\
& {\left[\text { for all } a_{-i}, b_{-i} \in X_{-i},\left(z_{i}, a_{-i}\right) \succsim\left(w_{i}, b_{-i}\right) \Rightarrow\left(x_{i}, a_{-i}\right) \succsim\left(y_{i}, b_{-i}\right)\right], } \\
&\left(x_{i}, y_{i}\right) \succsim_{i}^{* *}\left(z_{i}, w_{i}\right) \Leftrightarrow\left[\left(x_{i}, y_{i}\right) \succsim_{i}^{*}\left(z_{i}, w_{i}\right) \text { and }\left(w_{i}, z_{i}\right) \succsim_{i}^{*}\left(y_{i}, x_{i}\right)\right] .
\end{aligned}
$$

It is clear that the relations $\succsim_{i}^{*}$ and $\succsim_{i}^{* *}$ are always reflexive and transitive. Bouyssou and Pirlot (2005b, 2007) have shown that CR-AT are reflexive relations that are characterized by the fact that, for all $i \in N$, the relation $\succsim_{i}^{* *}$ is a weak order having at most three distinct equivalence classes and $\succsim_{i}^{ \pm}$ is a weak order. They have given necessary and sufficient conditions on $\succsim$ ensuring that this happens.

We are now in position to analyze the relations between CR-AT and RSRP. We start with a result showing that there are CR-AT that are not RSRP. This result exploits the fact that in a CR-AT the weak orders $\succsim_{i}^{ \pm}$ may have more than two distinct equivalence classes. This cannot be the case in a RSRP.

Lemma 15. A CR-AT may fail to be a reflexive RSRP.

Proof. This is obvious since a CR-AT does not impose a limit on the number of equivalence classes of the relations $\succsim_{i}^{ \pm}$. For instance, let $n=3, X_{1}=$ $X_{2}=X_{3}=\mathbb{R}$, and $X=\prod_{i=1}^{n} X_{i}$. For all $i \in N$, let $T_{i}$ be identical to $\geq$. Consider the relation $\succsim$ on $X$ such that

$$
x \succsim y \Leftrightarrow|T(x, y)| \geq|T(y, x)|
$$

By construction, this relation is a CR-AT. It is not a RSRP. Indeed, it is easy to see that for all $i \in N$, we have $\succsim_{i}^{ \pm}=\geq$. But we know that with a RSRP, all relations $\succsim_{i}^{ \pm}$have two distinct equivalence classes.

Our next result shows that a reflexive RSRP that is independent is a CR-AT. 
Lemma 16. A reflexive $R S R P$ that is independent is a CR-AT.

Proof. We know from Lemma 6 that in a RSRP all relations $\succsim_{i}^{ \pm}$are weak orders. Hence, it is enough to show that all relations $\succsim_{i}^{* *}$ are weak orders having at most three equivalence classes.

It is easy to show that, for all $i \in N$ and all $x_{i}, x_{i}^{\prime}, y_{i}, y_{i}^{\prime}, z_{i}, z_{i}^{\prime}, w_{i}, w_{i}^{\prime} \in X_{i}$, $\left(x_{i}, y_{i}\right) \succsim_{i}^{* *}\left(z_{i}, w_{i}\right), x_{i}^{\prime} \succsim_{i}^{ \pm} x_{i}, y_{i} \succsim_{i}^{ \pm} y_{i}^{\prime}, z_{i} \succsim_{i}^{ \pm} z_{i}^{\prime}, w_{i}^{\prime} \succsim_{i}^{ \pm} w_{i}$, imply $\left(x_{i}^{\prime}, y_{i}^{\prime}\right) \succsim_{i}^{* *}$ $\left(z_{i}^{\prime}, w_{i}^{\prime}\right)$ (Bouyssou and Pirlot 2005a, Lemma 3.8). In particular, using the reflexivity of $\succsim_{i}^{* *}$, if $x_{i} \sim_{i}^{ \pm} z_{i}$ and $y_{i} \sim_{i}^{ \pm} w_{i}$ we have $\left(x_{i}, y_{i}\right) \sim_{i}^{* *}\left(z_{i}, w_{i}\right)$.

Let $a_{i}, b_{i} \in X_{i}$. For the ordered pair $\left(a_{i}, b_{i}\right)$, it is clear that we have one of the following four situations:

1. $a_{i} \in \mathcal{A}_{i}$ and $b_{i} \in \mathcal{A}_{i}$,

2. $a_{i} \in \mathcal{A}_{i}$ and $b_{i} \notin \mathcal{A}_{i}$,

3. $a_{i} \notin \mathcal{A}_{i}$ and $b_{i} \in \mathcal{A}_{i}$,

4. $a_{i} \notin \mathcal{A}_{i}$ and $b_{i} \notin \mathcal{A}_{i}$.

Consider two ordered pairs $\left(x_{i}, y_{i}\right)$ and $\left(z_{i}, w_{i}\right)$ of elements in $X_{i}$. If these two ordered pairs are in the same situation, we have $x_{i} \sim_{i}^{ \pm} z_{i}$ and $y_{i} \sim_{i}^{ \pm} w_{i}$, so that $\left(x_{i}, y_{i}\right) \sim_{i}^{* *}\left(z_{i}, w_{i}\right)$.

All ordered pairs $\left(x_{i}, y_{i}\right)$ in the second situation are above all ordered pairs $\left(z_{i}, w_{i}\right)$ in the first situation in terms of $\succsim_{i}^{* *}$. Indeed, in this case, we know that $\left[\begin{array}{lllll}x_{i} & \sim_{i}^{ \pm} & z_{i} & \sim_{i}^{ \pm} & w_{i}\end{array}\right] \succ_{i}^{ \pm} y_{i}$. Since $\succsim_{i}^{* *}$ is reflexive, we have $\left(z_{i}, w_{i}\right) \succsim_{i}^{* *}\left(z_{i}, w_{i}\right)$. Using the fact that $x_{i} \succsim_{i}^{ \pm} z_{i}$ and $w_{i} \succsim_{i}^{ \pm} y_{i}$, we obtain $\left(x_{i}, y_{i}\right) \succsim_{i}^{* *}\left(z_{i}, w_{i}\right)$. A similar reasoning shows that all ordered pairs $\left(x_{i}, y_{i}\right)$ in the second situation are above all ordered pairs $\left(z_{i}, w_{i}\right)$ in the in the fourth situation in terms of $\succsim_{i}^{* *}$.

Similarly, it is easy to check that all ordered pairs $\left(x_{i}, y_{i}\right)$ in the first situation are above all ordered pairs $\left(z_{i}, w_{i}\right)$ in the in the third situation in terms of $\succsim_{i}^{* *}$ and that all ordered pairs $\left(x_{i}, y_{i}\right)$ in the fourth situation are above all ordered pairs $\left(z_{i}, w_{i}\right)$ in the in the third situation in terms of $\succsim_{i}^{* *}$.

Hence, the relation $\succsim_{i}^{* *}$ will be complete iff the elements in the first and fourth situations are equivalent in terms of $\succsim_{i}^{* *}$. Using Lemma 2.4, it is easy to see that this is equivalent to requiring that $\succsim$ is independent. Observe that, when this is the case, $\succsim_{i}^{* *}$ has at most three distinct equivalence classes.

Observe finally that an independent relation being a RSRP is a very particular CR-AT since, in this case, all relations $\succsim_{i}^{ \pm}$have only two distinct equivalence classes. 
Summarizing, there are CR-AT that are not reflexive RSRP (the ones in which the relations $\succsim_{i}^{ \pm}$have more than two equivalence classes) and there are reflexive RSRP that are not CR-AT (the ones that are not independent).

\section{The case of weak orders}

\subsection{Results}

Although all the conditions needed to characterize RSRP are independent in the class of all semiorders on $X$, the situation is vastly different when we turn to weak orders.

We know from Lemma 5 that conditions $A C 1_{i}, A C 2_{i}$, and $A C 3_{i}$ are equivalent for weak orders. The same is true for conditions $A C 1_{i}^{*}, A C 2_{i}^{*}$, $A C 3_{i}^{*}$ and $A C 4_{i}^{*}$.

Lemma 17. Let $\succsim$ be a weak order on a set $X$. Then conditions $A C 1_{i}^{*}$, $A C 2_{i}^{*}, A C 3_{i}^{*}$ and $A C 4_{i}^{*}$ are equivalent.

Proof. $\quad\left[A C 2_{i}^{*} \Rightarrow A C 1_{i}^{*}\right]$ Suppose that $A C 1_{i}^{*}$ is violated, so that $\left(x_{i}, a_{-i}\right) \succsim c$, $\left(y_{i}, b_{-i}\right) \succsim d,\left(y_{i}, a_{-i}\right) \nsucceq c,\left(z_{i}, b_{-i}\right) \nsucceq d$. Using the fact that $\succsim$ is a weak order, we obtain $\left(x_{i}, a_{-i}\right) \succ\left(y_{i}, a_{-i}\right)$ and $\left(y_{i}, b_{-i}\right) \succ\left(z_{i}, b_{-i}\right)$. Since $\succsim$ is reflexive, we have $\left(y_{i}, a_{-i}\right) \succsim\left(y_{i}, a_{-i}\right)$ and $\left(z_{i}, b_{-i}\right) \succsim\left(z_{i}, b_{-i}\right)$. Applying $A C 2_{i}^{*}$, we obtain $\left(y_{i}, a_{-i}\right) \succsim\left(x_{i}, a_{-i}\right)$ or $\left(z_{i}, b_{-i}\right) \succsim\left(y_{i}, b_{-i}\right)$, a contradiction.

$\left[A C 3_{i}^{*} \Rightarrow A C 2_{i}^{*}\right]$ Suppose that $A C 2_{i}^{*}$ is violated, so that $c \succsim\left(y_{i}, a_{-i}\right)$, $d \succsim\left(z_{i}, b_{-i}\right), c \nsucceq\left(x_{i}, a_{-i}\right)$, and $d \nsucceq\left(y_{i}, b_{-i}\right)$. Using the fact that $\succsim$ is a weak order, we obtain $\left(x_{i}, a_{-i}\right) \succ\left(y_{i}, a_{-i}\right)$ and $\left(y_{i}, b_{-i}\right) \succ\left(z_{i}, b_{-i}\right)$. Since $\succsim$ is reflexive, we have $\left(x_{i}, a_{-i}\right) \succsim\left(x_{i}, a_{-i}\right)$ and $\left(z_{i}, b_{-i}\right) \succsim\left(z_{i}, b_{-i}\right)$. Applying $A C 3_{i}^{*}$, we obtain $\left(y_{i}, a_{-i}\right) \succsim\left(x_{i}, a_{-i}\right)$ or $\left(z_{i}, b_{-i}\right) \succsim\left(y_{i}, b_{-i}\right)$, a contradiction.

$\left[A C 4_{i}^{*} \Rightarrow A C 3_{i}^{*}\right]$ Suppose that $A C 3_{i}^{*}$ is violated, so that $\left(x_{i}, a_{-i}\right) \succsim c$, $d \succsim\left(z_{i}, b_{-i}\right),\left(y_{i}, a_{-i}\right) \succsim c, d \nsucceq\left(y_{i}, b_{-i}\right)$. Using the fact that $\succsim$ is a weak order, we obtain $\left(y_{i}, b_{-i}\right) \succ\left(z_{i}, b_{-i}\right)$ and $\left(x_{i}, a_{-i}\right) \succ\left(y_{i}, a_{-i}\right)$. Since $\succsim$ is reflexive, we have $\left(y_{i}, a_{-i}\right) \succsim\left(y_{i}, a_{-i}\right)$ and $\left(y_{i}, b_{-i}\right) \succsim\left(y_{i}, b_{-i}\right)$. Applying $A C 4_{i}^{*}$, we obtain $\left(y_{i}, a_{-i}\right) \succsim\left(x_{i}, a_{-i}\right)$ or $\left(z_{i}, b_{-i}\right) \succsim\left(y_{i}, b_{-i}\right)$, a contradiction.

$\left[A C 1_{i}^{*} \Rightarrow A C 4_{i}^{*}\right]$ Suppose that $A C 4_{i}^{*}$ is violated, so that $c \succsim\left(y_{i}, a_{-i}\right)$, $\left(y_{i}, b_{-i}\right) \succsim d, c \succsim\left(x_{i}, a_{-i}\right),\left(z_{i}, b_{-i}\right) \succsim d$. Using the fact that $\succsim$ is a weak order, we obtain $\left(x_{i}, a_{-i}\right) \succ\left(y_{i}, a_{-i}\right)$ and $\left(y_{i}, b_{-i}\right) \succ\left(z_{i}, b_{-i}\right)$. Since $\succsim$ is reflexive, we have $\left(x_{i}, a_{-i}\right) \succsim\left(x_{i}, a_{-i}\right)$ and $\left(y_{i}, b_{-i}\right) \succsim\left(y_{i}, b_{-i}\right)$. Applying $A C 1_{i}^{*}$, we obtain $\left(y_{i}, a_{-i}\right) \succsim\left(x_{i}, a_{-i}\right)$ or $\left(z_{i}, b_{-i}\right) \succsim\left(y_{i}, b_{-i}\right)$, a contradiction. 
We have shown above that $A C 1_{i}^{*}$ (resp. $\left.A C 2_{i}^{*}, A C 3_{i}^{*}, A C 4_{i}^{*}\right)$ was equivalent to the conjunction of $A C 1_{i}$ and $A C 1_{i}^{* *}$ (resp. $A C 2_{i}$ and $A C 2_{i}^{* *}, A C 3_{i}$ and $A C 3_{i}^{* *}, A C 3_{i}$ and $\left.A C 4_{i}^{* *}\right)$. In the class of weak orders, conditions $A C 1_{i}$ and $A C 1_{i}^{* *}$ are independent.

Lemma 18. In the class of all weak orders on $X$, conditions $A C 1_{i}$ and $A C 1_{i}^{* *}$ are independent.

Proof. See Appendix C.

Summarizing the above observations, we have:

Proposition 3. Let $\succsim$ be a weak order on $X$. It is a Relation with a Single Reference Point iff it satisfies $A C 1^{*}$ iff it satisfies $A C 1$ and $A C 1^{* *}$.

On the basis of Lemmas 5, 13 and 17 and building obvious variations of Lemma 18, it is possible to formulate many alternative equivalent results for the case of weak orders. We leave the details to the interested reader.

\subsection{Relation to models based on a discrete Sugeno integral}

We have shown in Section 3 that a RSRP that is a weak order has a numerical representation such that:

$$
x \succsim y \Leftrightarrow \mu(\mathcal{S}(x)) \geq \mu(\mathcal{S}(y))
$$

where $\mu$ is a normalized capacity on $N$.

This representation is reminiscent of representation of weak orders based on a discrete Sugeno integral studied in Bouyssou et al. (2009) and Greco et al. (2004). Indeed, as shown below, a RSRP always has a representation using a discrete Sugeno integral.

The following definitions are taken from Bouyssou et al. (2009).

Definition 9. Let $\beta=\left(\beta_{1}, \beta_{2}, \ldots, \beta_{p}\right) \in[0,1]^{p}$. The discrete Sugeno integral of the vector $\left(\beta_{1}, \beta_{2}, \ldots, \beta_{p}\right) \in[0,1]^{p}$ w.r.t. the normalized capacity $\nu$ on $P=\{1,2, \ldots, p\}$ is defined by:

$$
\operatorname{Sug}_{\nu}[\beta]=\bigvee_{T \subseteq P}\left[\nu(T) \wedge\left(\bigwedge_{i \in T} \beta_{i}\right)\right]
$$


Definition 10. A weak order $\succsim$ on $X$ has a representation in the discrete Sugeno integral model if there are a normalized capacity $\mu$ on $N$ and functions $u_{i}: X_{i} \rightarrow[0,1]$ such that, for all $x, y \in X$,

$x \succsim y \Leftrightarrow \operatorname{Sug}_{\mu}\left[\left(u_{1}\left(x_{1}\right), u_{2}\left(x_{2}\right), \ldots, u_{n}\left(x_{n}\right)\right)\right] \geq \operatorname{Sug}_{\mu}\left[\left(u_{1}\left(y_{1}\right), u_{2}\left(y_{2}\right), \ldots, u_{n}\left(y_{n}\right)\right)\right]$.

Definition 11. The relation $\succsim$ on $X$ is said to be strongly 2-graded on attribute $i \in N$ (condition $2^{*}$-graded ${ }_{i}$ ) if, for all $a, b, c, d \in X$ and all $x_{i}, y_{i}, z_{i} \in$ $X_{i}$

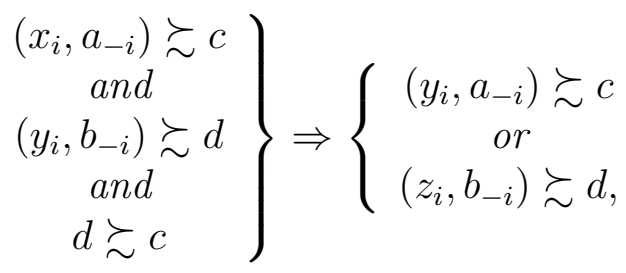

The relation $\succsim$ on $X$ is said to be 2-graded on attribute $i \in N$ (condition 2-graded $)$ if, for all $a, b, c, d \in X$ and all $x_{i}, y_{i}, z_{i} \in X_{i}$,

$$
\left.\begin{array}{c}
\left(x_{i}, a_{-i}\right) \succsim c \\
\text { and } \\
\left(y_{i}, b_{-i}\right) \succsim d \\
\text { and } \\
\left(x_{i}, b_{-i}\right) \succsim d \\
\text { and } \\
d \succsim c
\end{array}\right\} \Rightarrow\left\{\begin{array}{c}
\left(y_{i}, a_{-i}\right) \succsim c \\
\text { or } \\
\left(z_{i}, b_{-i}\right) \succsim d,
\end{array}\right.
$$

A binary relation is said to be strongly 2 -graded (condition $2^{*}$-graded) if it is strongly 2-graded on all attributes $i \in N$. Similarly, a binary relation is said to be 2-graded (condition 2-graded) if it is 2-graded on all attributes $i \in N$.

Bouyssou et al. (2009, Lemma 1) have shown that condition $2^{*}$-graded $_{i}$ holds iff conditions 2 -graded $i$ and $A C 1_{i}$ hold.

It is clear that condition $2^{*}-\operatorname{graded}_{i}$ is a weakening of $A C 1_{i}^{*}$ since it amounts to adding a premise to this condition. Similarly, condition 2-graded $i$ is a weakening of $A C 1_{i}^{* *}$. Bouyssou et al. (2009) and Greco et al. (2004) have shown the following (this result can be extended to cover sets of arbitrary cardinality adding a condition imposing that the weak order has a numerical representation. This will not be useful here). 
Proposition 4. Let $\succsim$ be a weak order on $X$ such that $X / \sim$ is at most countably infinite. Then $\succsim$ has a representation in the discrete Sugeno integral model iff it satisfies condition $2^{*}$-graded iff it satisfies conditions $A C 1$ and 2-graded.

The following lemma is a direct consequence of the fact that condition $2^{*}$ graded $_{i}$ is a weakening of $A C 1_{i}^{*}$.

Lemma 19. Let $\succsim$ be a weak order. If it is a RSRP, then it has a representation in the discrete Sugeno integral model.

Proof. We know that a RSRP that is weak order only has a finite number of distinct equivalence classes. Hence, the proof follows from Proposition 4 and the fact that $A C 1^{*}$ implies $2^{*}$-graded.

The above lemma has shown that, for weak orders, a RSRP always has a representation in the discrete Sugeno integral model. Suppose that the weak order $\succsim$ is represented as a RSRP using sets $\mathcal{A}_{i}$ and an importance relation $\unrhd$. We know from Lemma 2 that $\unrhd$ is a weak order on $N$, so that it can be represented by a normalized capacity $\mu$ on $N$. It is then easy to devise a representation of this weak order in the discrete Sugeno integral model.

Define, for all $i \in N$ and all $x_{i} \in X_{i}$,

$$
u_{i}\left(x_{i}\right)= \begin{cases}1 & \text { if } x_{i} \in \mathcal{A}_{i} \\ 0 & \text { otherwise }\end{cases}
$$

Using such functions $u_{i}$, it is easy to see that, for all $x \in X$, and all $J \subseteq N$, we have:

$$
\mu(J) \wedge\left(\bigwedge_{i \in J} u_{i}\left(x_{i}\right)\right)= \begin{cases}\mu(J) & \text { if } x_{i} \in \mathcal{A}_{i} \text { for all } i \in J \\ 0 & \text { otherwise. }\end{cases}
$$

Hence, when $\mathcal{S}(x)=J$ we have $S u g_{\mu}\left[\left(u_{1}\left(x_{1}\right), u_{2}\left(x_{2}\right), \ldots, u_{n}\left(x_{n}\right)\right)\right]=\mu(J)$, so that

$$
\begin{aligned}
x \succsim y & \Leftrightarrow \mathcal{S}(x) \unrhd \mathcal{S}(y) \\
& \Leftrightarrow \mu(\mathcal{S}(x)) \geq \mu(\mathcal{S}(y)) \\
& \Leftrightarrow S u g_{\mu}\left[\left(u_{1}\left(x_{1}\right), u_{2}\left(x_{2}\right), \ldots, u_{n}\left(x_{n}\right)\right)\right] \geq S u g_{\mu}\left[\left(u_{1}\left(y_{1}\right), u_{2}\left(y_{2}\right), \ldots, u_{n}\left(y_{n}\right)\right)\right] .
\end{aligned}
$$

We will see in Appendix D that the situation is different when considering relations using several reference points: such relations may not have a representation in the discrete Sugeno integral model. 


\section{Discussion}

This paper has shown how to use the general model developed in Bouyssou and Pirlot (2004b) to characterize preference models using a single reference point introduced by Rolland (2003, 2006a,b, 2008). This analysis is extended to the case of multiple reference points in Appendix D. Basically, models using reference points are particular cases of the model inducing complete traces on the levels of each attribute. The number of reference points is linked to the number of distinct equivalence classes generated by these traces. Models using a single reference point generate traces that are quite rough. We have proposed a complete characterization of these models using a traditional conjoint measurement setting in which the only primitive is a preference relation $\succsim$ on $X$. This analysis leads to conditions that could be the subject of empirical tests. Moreover these conditions allow to better position models using reference points among other preference models for multiattributed alternatives. In particular, we have shown that preference models using a single reference point that are weak orders are a particular case of a model based on the discrete Sugeno integral. Moreover we have analyzed the relations between preference models using a single reference point and concordance relations. None of these two models is a subclass of the other.

We conclude with the indication of directions for future research on the subject. The first is to analyze models using reference points that would include an idea of "non-discordance" as in the ELECTRE TRI model. Using the results in Bouyssou and Marchant (2007a,b), this should not be overly difficult. The second is to pursue the analysis of preference models using several reference points. Indeed, Rolland (2003, 2006a,b, 2008) has proposed several particular cases of the general model that we study in Appendix D. Finally, it is clearly of vital interest to investigate elicitation techniques that would lead to specify the parameters of the models that we have studied.

\section{Appendices}

In all examples in Sections $\mathrm{A}, \mathrm{B}$, and $\mathrm{C}$, we take $n=3$ and $X_{1}=\{x, y, z\}$, $X_{2}=\{a, b\}$ and $X_{3}=\{p, q\}$. To save space, we often write $x a p$ instead of $(x, a, p)$. 


\section{A. Proof of Lemma 12}

We have to show that in the class of all semiorders on $X$, conditions $A C 1^{*}, A C 2^{*}, A C 3^{*}$, and $A C 4^{*}$ are independent.

We provide below the required four examples. In all these examples, we define $\succsim$ by its boolean matrix, so that it is easy to check that the relations considered below are semiorders. Indeed, if it is possible to arrange the rows and columns of the boolean matrix in the same order so that the boolean matrix is stepped, we know that the relation is a semiorder (Aleskerov et al. 2007).

Example 1. Let $\succsim$ on $X$ be defined by the following table:

\begin{tabular}{ccccccccccccc}
\hline & $x a q$ & $y a q$ & $z a q$ & $x a p$ & $y a p$ & $z a p$ & $y b q$ & $x b p$ & $x b q$ & $y b p$ & $z b p$ & $z b q$ \\
$x a q$ & 1 & 1 & 1 & 1 & 1 & 1 & 1 & 1 & 1 & 1 & 1 & 1 \\
$y a q$ & 1 & 1 & 1 & 1 & 1 & 1 & 1 & 1 & 1 & 1 & 1 & 1 \\
$z a q$ & 1 & 1 & 1 & 1 & 1 & 1 & 1 & 1 & 1 & 1 & 1 & 1 \\
$x a p$ & 1 & 1 & 1 & 1 & 1 & 1 & 1 & 1 & 1 & 1 & 1 & 1 \\
$y a p$ & 1 & 1 & 1 & 1 & 1 & 1 & 1 & 1 & 1 & 1 & 1 & 1 \\
$z a p$ & 1 & 1 & 1 & 1 & 1 & 1 & 1 & 1 & 1 & 1 & 1 & 1 \\
$y b q$ & 1 & 1 & 1 & 1 & 1 & 1 & 1 & 1 & 1 & 1 & 1 & 1 \\
$x b p$ & 1 & 1 & 1 & 1 & 1 & 1 & 1 & 1 & 1 & 1 & 1 & 1 \\
$x b q$ & 1 & 1 & 1 & 1 & 1 & 1 & 1 & 1 & 1 & 1 & 1 & 1 \\
$y b p$ & 0 & 0 & 0 & 1 & 1 & 1 & 1 & 1 & 1 & 1 & 1 & 1 \\
$z b p$ & 0 & 0 & 0 & 0 & 0 & 0 & 1 & 1 & 1 & 1 & 1 & 1 \\
$z b q$ & 0 & 0 & 0 & 0 & 0 & 0 & 1 & 1 & 1 & 1 & 1 & 1 \\
\hline
\end{tabular}

It is clear that this relation is a semiorder on $X$.

It is easy to check that we have $a \succ_{2}^{+} b, a \succ_{2}^{-} b, q \succ_{3}^{+} p$ and $q \succ_{3}^{-} p$. Using Lemma 9 shows that $A C 1_{i}^{*}, A C 2_{i}^{*}, A C 3_{i}^{*}$, and $A C 4_{i}^{*}$ hold for $i=2,3$.

On attribute 1 , we have $x \sim_{1}^{-} y \sim_{1}^{-} z$ and $x \succ_{1}^{+} y \succ_{1}^{+} z$. Using Lemma 9 shows that $A C 2_{1}^{*}, A C 3_{1}^{*}$ and $A C 4_{1}^{*}$, while $A C 1_{1}^{*}$ is clearly violated.

Transposing the boolean matrix in the above example, one easily obtain an example satisfying all our conditions except $A C 2$ on one attribute. Indeed, on each attribute the consequence of this transposition is is to interchange the roles of $\succsim_{i}^{+}$and $\succsim_{i}^{-}$and to reverse them. This is detailed below.

Example 2. Let $\succsim$ on $X$ be defined by the following table: 


\begin{tabular}{ccccccccccccc}
\hline & $z b q$ & $z b p$ & $y b p$ & $x b q$ & $x b p$ & $y b q$ & $z a p$ & $y a p$ & $x a p$ & $z a q$ & $y a q$ & $x a q$ \\
$z b q$ & 1 & 1 & 1 & 1 & 1 & 1 & 1 & 1 & 1 & 1 & 1 & 1 \\
$z b p$ & 1 & 1 & 1 & 1 & 1 & 1 & 1 & 1 & 1 & 1 & 1 & 1 \\
$y b p$ & 1 & 1 & 1 & 1 & 1 & 1 & 1 & 1 & 1 & 1 & 1 & 1 \\
$x b q$ & 1 & 1 & 1 & 1 & 1 & 1 & 1 & 1 & 1 & 1 & 1 & 1 \\
$x b p$ & 1 & 1 & 1 & 1 & 1 & 1 & 1 & 1 & 1 & 1 & 1 & 1 \\
$y b q$ & 1 & 1 & 1 & 1 & 1 & 1 & 1 & 1 & 1 & 1 & 1 & 1 \\
$z a p$ & 0 & 0 & 1 & 1 & 1 & 1 & 1 & 1 & 1 & 1 & 1 & 1 \\
$y a p$ & 0 & 0 & 1 & 1 & 1 & 1 & 1 & 1 & 1 & 1 & 1 & 1 \\
$x a p$ & 0 & 0 & 1 & 1 & 1 & 1 & 1 & 1 & 1 & 1 & 1 & 1 \\
$z a q$ & 0 & 0 & 0 & 1 & 1 & 1 & 1 & 1 & 1 & 1 & 1 & 1 \\
$y a q$ & 0 & 0 & 0 & 1 & 1 & 1 & 1 & 1 & 1 & 1 & 1 & 1 \\
$x a q$ & 0 & 0 & 0 & 1 & 1 & 1 & 1 & 1 & 1 & 1 & 1 & 1 \\
\hline
\end{tabular}

It is clear that this relation is a semiorder on $X$.

It is easy to check that we have $b \succ_{2}^{+} a, b \succ_{2}^{-} a, p \succ_{3}^{+} q$ and $p \succ_{3}^{-} q$. Using Lemma 9 shows that $A C 1_{i}^{*}, A C 2_{i}^{*}, A C 3_{i}^{*}$, and $A C 4_{i}^{*}$ hold for $i=2,3$.

On attribute 1 , we have $x \sim_{1}^{+} y \sim_{1}^{+} z$ and $z \succ_{1}^{-} y \succ_{1}^{-} x$. Using Lemma 9 shows that $A C 1_{1}^{*}, A C 3_{1}^{*}$ and $A C 4_{1}^{*}$, while $A C 2_{1}^{*}$ is clearly violated.

Example 3 . Let $\succsim$ on $X$ be defined by the following table:

\begin{tabular}{ccccccccccccc}
\hline & $x a q$ & $y a q$ & $x a p$ & $x b p$ & $x b q$ & $y a p$ & $y b q$ & $z a p$ & $z a q$ & $z b q$ & $y b p$ & $z b p$ \\
$x a q$ & 1 & 1 & 1 & 1 & 1 & 1 & 1 & 1 & 1 & 1 & 1 & 1 \\
$y a q$ & 1 & 1 & 1 & 1 & 1 & 1 & 1 & 1 & 1 & 1 & 1 & 1 \\
$x a p$ & 1 & 1 & 1 & 1 & 1 & 1 & 1 & 1 & 1 & 1 & 1 & 1 \\
$x b p$ & 1 & 1 & 1 & 1 & 1 & 1 & 1 & 1 & 1 & 1 & 1 & 1 \\
$x b q$ & 1 & 1 & 1 & 1 & 1 & 1 & 1 & 1 & 1 & 1 & 1 & 1 \\
$y a p$ & 1 & 1 & 1 & 1 & 1 & 1 & 1 & 1 & 1 & 1 & 1 & 1 \\
$y b q$ & 1 & 1 & 1 & 1 & 1 & 1 & 1 & 1 & 1 & 1 & 1 & 1 \\
$z a p$ & 1 & 1 & 1 & 1 & 1 & 1 & 1 & 1 & 1 & 1 & 1 & 1 \\
$z a q$ & 1 & 1 & 1 & 1 & 1 & 1 & 1 & 1 & 1 & 1 & 1 & 1 \\
$z b q$ & 1 & 1 & 1 & 1 & 1 & 1 & 1 & 1 & 1 & 1 & 1 & 1 \\
$y b p$ & 0 & 0 & 1 & 1 & 1 & 1 & 1 & 1 & 1 & 1 & 1 & 1 \\
$z b p$ & 0 & 0 & 1 & 1 & 1 & 1 & 1 & 1 & 1 & 1 & 1 & 1 \\
\hline
\end{tabular}

It is clear that this relation is a semiorder on $X$.

It is easy to check that we have $a \succ_{2}^{+} b, a \succ_{2}^{-} b, q \succ_{3}^{+} p$ and $q \succ_{3}^{-} p$. Using Lemma 9 shows that $A C 1_{i}^{*}, A C 2_{i}^{*}, A C 3_{i}^{*}$, and $A C 4_{i}^{*}$ hold for $i=2,3$. 
On attribute 1 , we have $x \succ_{1}^{+}\left[\begin{array}{lll}y \sim_{1}^{+} & z\end{array}\right]$ and $\left[\begin{array}{lll}x \sim_{1}^{-} & y\end{array}\right] \succ_{1}^{-} z$. Using Lemma 9 shows that $A C 1_{1}^{*}, A C 2_{1}^{*}$ and $A C 4_{1}^{*}$, while $A C 3_{1}^{*}$ is clearly violated.

Example 4 . Let $\succsim$ on $X$ be defined by the following table:

\begin{tabular}{ccccccccccccc}
\hline & $x b p$ & $x b q$ & $x a p$ & $x a q$ & $y a p$ & $y a q$ & $y b p$ & $y b q$ & $z a q$ & $z b q$ & $z a p$ & $z b p$ \\
$x b p$ & 1 & 1 & 1 & 1 & 1 & 1 & 1 & 1 & 1 & 1 & 1 & 1 \\
$x b q$ & 1 & 1 & 1 & 1 & 1 & 1 & 1 & 1 & 1 & 1 & 1 & 1 \\
$x a p$ & 1 & 1 & 1 & 1 & 1 & 1 & 1 & 1 & 1 & 1 & 1 & 1 \\
$x a q$ & 1 & 1 & 1 & 1 & 1 & 1 & 1 & 1 & 1 & 1 & 1 & 1 \\
$y a p$ & 1 & 1 & 1 & 1 & 1 & 1 & 1 & 1 & 1 & 1 & 1 & 1 \\
$y a q$ & 1 & 1 & 1 & 1 & 1 & 1 & 1 & 1 & 1 & 1 & 1 & 1 \\
$y b p$ & 1 & 1 & 1 & 1 & 1 & 1 & 1 & 1 & 1 & 1 & 1 & 1 \\
$y b q$ & 1 & 1 & 1 & 1 & 1 & 1 & 1 & 1 & 1 & 1 & 1 & 1 \\
$z a q$ & 1 & 1 & 1 & 1 & 1 & 1 & 1 & 1 & 1 & 1 & 1 & 1 \\
$z b q$ & 1 & 1 & 1 & 1 & 1 & 1 & 1 & 1 & 1 & 1 & 1 & 1 \\
$z a p$ & 0 & 0 & 1 & 1 & 1 & 1 & 1 & 1 & 1 & 1 & 1 & 1 \\
$z b p$ & 0 & 0 & 1 & 1 & 1 & 1 & 1 & 1 & 1 & 1 & 1 & 1 \\
\hline
\end{tabular}

It is clear that this relation is a semiorder on $X$.

It is easy to check that we have $b \sim_{2}^{+} a, b \succ_{2}^{-} a, q \succ_{3}^{+} p$ and $q \sim_{3}^{-} p$. Using Lemma 9 shows that $A C 1_{i}^{*}, A C 2_{i}^{*}, A C 3_{i}^{*}$, and $A C 4_{i}^{*}$ hold for $i=2,3$.

On attribute 1 , we have $\left[x \sim_{1}^{+} y\right] \succ_{1}^{+} z$ and $x \succ_{1}^{-}\left[\begin{array}{lll}y \sim_{1}^{-} & z\end{array}\right]$. Using Lemma 9 shows that $A C 1_{1}^{*}, A C 2_{1}^{*}$ and $A C 3_{1}^{*}$, while $A C 4_{1}^{*}$ is clearly violated.

Observe that going from Example 3 to Example 4 does not amount to transposing the boolean matrix of the relation in Example 3. Transposing a relation violating only $A C 3_{1}^{*}$ does not lead to a relation violating only $A C 4_{1}^{*}$.

\section{B. Proof of Lemma 14}

We have to show that in the class of all semiorders on $X$, conditions $A C 1_{i}, A C 2_{i}, A C 3_{i}, A C 1^{* *}, A C 2^{* *}, A C 3^{* *}$, and $A C 4^{* *}$ are independent. Seven examples are needed to do so.

Observe first that in Examples 1-4, conditions $A C 1, A C 2, A C 3$ are satisfied. Hence, each of these examples violates exactly one of $A C 1_{i}^{* *}, A C 2_{i}^{* *}$, $A C 3_{i}^{* *}$, and $A C 4_{i}^{* *}$. It remains to find three more examples.

In order to check the following three examples, it will be helpful to observe that whenever $X_{i}$ has only two elements, conditions $A C 1_{i}^{* *}, A C 2_{i}^{* *}, A C 3_{i}^{* *}$, 
and $A C 4_{i}^{* *}$ are always satisfied. Indeed, the premises of $A C 1_{i}^{* *}$ state that $\left(x_{i}, a_{-i}\right) \succsim c,\left(y_{i}, b_{-i}\right) \succsim d$, and $\left(x_{i}, b_{-i}\right) \succsim d$. The two possible conclusions of $A C 1_{i}^{* *}$ are $\left(y_{i}, a_{-i}\right) \succsim c$ or $\left(z_{i}, b_{-i}\right) \succsim d$. If $x_{i}=y_{i}$ then the first conclusion of $A C 1_{i}^{* *}$ trivially holds. If $x_{i} \neq y_{i}$ then the second possible conclusion of $A C 1_{i}^{* *}$ will trivially hold since $z_{i}$ must be either $x_{i}$ or $y_{i}$. A similar reasoning applies to $A C 2_{i}^{* *}$ and $A C 4_{i}^{* *}$. The premises of $A C 3_{i}^{* *}$ state that $\left(x_{i}, a_{-i}\right) \succsim c$, $d \succsim\left(z_{i}, b_{-i}\right)$. The three possible conclusions are $\left(y_{i}, a_{-i}\right) \succsim c, d \succsim\left(y_{i}, b_{-i}\right)$, or $d \succsim\left(x_{i}, b_{-i}\right)$. If $x_{i}=z_{i}$, then the third conclusion of $A C 3_{i}^{* *}$ trivially holds. If $x_{i} \neq z_{i}$, then the $y_{i}$ in the conclusion of $A C 3_{i}^{* *}$ must be either $x_{i}$ or $z_{i}$. In the first (resp. second) case, the first (resp. second) conclusion trivially holds.

Example 5. Let $\succsim$ on $X$ be defined by the following table:

\begin{tabular}{lcccccccccccc}
\hline & $z b p$ & $y b p$ & $x b p$ & $y b q$ & $z b q$ & $x b q$ & $z a p$ & $y a p$ & $x a p$ & $y a q$ & $z a q$ & $x a q$ \\
$z b p$ & 1 & 1 & 1 & 1 & 1 & 1 & 1 & 1 & 1 & 1 & 1 & 1 \\
$y b p$ & 1 & 1 & 1 & 1 & 1 & 1 & 1 & 1 & 1 & 1 & 1 & 1 \\
$x b p$ & 1 & 1 & 1 & 1 & 1 & 1 & 1 & 1 & 1 & 1 & 1 & 1 \\
$y b q$ & 1 & 1 & 1 & 1 & 1 & 1 & 1 & 1 & 1 & 1 & 1 & 1 \\
$z b q$ & 0 & 0 & 0 & 1 & 1 & 1 & 1 & 1 & 1 & 1 & 1 & 1 \\
$x b q$ & 0 & 0 & 0 & 1 & 1 & 1 & 1 & 1 & 1 & 1 & 1 & 1 \\
$z a p$ & 0 & 0 & 0 & 1 & 1 & 1 & 1 & 1 & 1 & 1 & 1 & 1 \\
$y a p$ & 0 & 0 & 0 & 0 & 0 & 0 & 1 & 1 & 1 & 1 & 1 & 1 \\
$x a p$ & 0 & 0 & 0 & 0 & 0 & 0 & 1 & 1 & 1 & 1 & 1 & 1 \\
$y a q$ & 0 & 0 & 0 & 0 & 0 & 0 & 1 & 1 & 1 & 1 & 1 & 1 \\
$z a q$ & 0 & 0 & 0 & 0 & 0 & 0 & 0 & 0 & 0 & 1 & 1 & 1 \\
$x a q$ & 0 & 0 & 0 & 0 & 0 & 0 & 0 & 0 & 0 & 1 & 1 & 1 \\
\hline
\end{tabular}

It is clear that this relation is a semiorder.

It is easy to check that the traces are as follows.

On attribute 2, we have $b \succ_{2}^{+} a$ and $b \succ_{2}^{-} a$. Hence, $A C 1_{2}, A C 2_{2}$, and $A C 3_{2}$ hold. Since $X_{2}$ has only two elements, $A C 1_{2}^{* *}, A C 2_{2}^{* *}, A C 3_{2}^{* *}$, and $A C 4_{2}^{* *}$ trivially hold.

On attribute 3 , we have $p \succ_{3}^{+} q$ and $p \succ_{3}^{-} q$. Hence, $A C 1_{3}, A C 2_{3}$, and $A C 3_{3}$ hold. Since $X_{3}$ has only two elements, $A C 1_{3}^{* *}, A C 2_{3}^{* *}, A C 3_{3}^{* *}$, and $A C 4_{3}^{* *}$ trivially hold.

On attribute $1, \succsim_{1}^{-}$is a clique. Furthermore, we have $y \succ_{1}^{+} x$ and $z \succ_{1}^{+} x$, but neither $y \succsim_{1}^{+} z$ nor $z \succsim_{1}^{+} y$. It easy to check that this implies that $A C 2_{1}$ and $A C 3_{1}$ hold, while $A C 1_{1}$ is violated. 
It remains to check that $A C 1_{1}^{* *}, A C 2_{1}^{* *}, A C 3_{1}^{* *}$, and $A C 4_{1}^{* *}$ are satisfied.

Using Lemma 9 , it is easy to check that $A C 2_{1}^{*}, A C 3_{1}^{*}$, and $A C 4_{1}^{*}$ are satisfied. Indeed, since $\succsim_{1}^{-}$is a clique, the premise of $A C 2_{1}^{*}$ and $A C 4_{1}^{*}$ is never satisfied while the conclusion $A C 3_{1}^{*}$ always holds.

Let us check that $A C 1_{1}^{* *}$ holds.

The second premise of $A C 1_{1}^{* *}$ is $\left(y_{1}, b_{-1}\right) \succsim d$. If $y_{1}=x$ then since all elements of $X_{1}$ are above $x$ according to $\succsim_{1}^{+}$, we will have that $\left(z_{1}, b_{-1}\right) \succsim d$, for all $z_{1} \in X_{1}$.

Similarly, the first premise of $A C 1_{1}^{* *}$ is $\left(x_{1}, a_{-1}\right) \succsim c$. If $x_{1}=x$ then since all elements of $X_{1}$ are above $x$ according to $\succsim_{1}^{+}$, we will have that $\left(y_{1}, a_{-1}\right) \succsim c$, for all $y_{1} \in X_{1}$.

This shows that the only possible violations of $A C 1_{1}^{* *}$ will occur if either $x_{1}=y$ and $y_{1}=z$ or $x_{1}=z$ and $y_{1}=y$.

Let us consider the first case with $x_{1}=y$ and $y_{1}=z$. The premises of $A C 1_{1}^{* *}$ state that $\left(y, a_{-1}\right) \succsim c,\left(z, b_{-1}\right) \succsim d$, and $\left(y, b_{-1}\right) \succsim d$. It is easy to check that whenever we have both $\left(z, b_{-1}\right) \succsim d$ and $\left(y, b_{-1}\right) \succsim d$, we also have that $\left(x, b_{-1}\right) \succsim d$. Hence the second conclusion of $A C 1_{1}^{* *}$ is satisfied.

Let us now consider the second case in which $x_{1}=z$ and $y_{1}=y$. The premises of $A C 1_{1}^{* *}$ state that $\left(z, a_{-1}\right) \succsim c,\left(y, b_{-1}\right) \succsim d$, and $\left(z, b_{-1}\right) \succsim d$. As observed above, whenever we have both $\left(y, b_{-1}\right) \succsim d$ and $\left(z, b_{-1}\right) \succsim d$, we also have that $\left(x, b_{-1}\right) \succsim d$. Hence the second conclusion of $A C 1_{1}^{* *}$ is satisfied.

Hence $A C 1_{1}^{* *}$ holds.

Transposing the boolean matrix in the above example, one easily obtain an example satisfying all our conditions except $A C 2$ on one attribute. This is detailed below.

Example 6 . Let $\succsim$ on $X$ be defined by the following table: 


\begin{tabular}{ccccccccccccc}
\hline & $x a q$ & $z a q$ & $y a q$ & $x a p$ & $y a p$ & $z a p$ & $x b q$ & $z b q$ & $y b q$ & $x b p$ & $y b p$ & $z b p$ \\
$x a q$ & 1 & 1 & 1 & 1 & 1 & 1 & 1 & 1 & 1 & 1 & 1 & 1 \\
$z a q$ & 1 & 1 & 1 & 1 & 1 & 1 & 1 & 1 & 1 & 1 & 1 & 1 \\
$y a q$ & 1 & 1 & 1 & 1 & 1 & 1 & 1 & 1 & 1 & 1 & 1 & 1 \\
$x a p$ & 0 & 0 & 1 & 1 & 1 & 1 & 1 & 1 & 1 & 1 & 1 & 1 \\
$y a p$ & 0 & 0 & 1 & 1 & 1 & 1 & 1 & 1 & 1 & 1 & 1 & 1 \\
$z a p$ & 0 & 0 & 1 & 1 & 1 & 1 & 1 & 1 & 1 & 1 & 1 & 1 \\
$x b q$ & 0 & 0 & 0 & 0 & 0 & 1 & 1 & 1 & 1 & 1 & 1 & 1 \\
$z b q$ & 0 & 0 & 0 & 0 & 0 & 1 & 1 & 1 & 1 & 1 & 1 & 1 \\
$y b q$ & 0 & 0 & 0 & 0 & 0 & 1 & 1 & 1 & 1 & 1 & 1 & 1 \\
$x b p$ & 0 & 0 & 0 & 0 & 0 & 0 & 0 & 0 & 1 & 1 & 1 & 1 \\
$y b p$ & 0 & 0 & 0 & 0 & 0 & 0 & 0 & 0 & 1 & 1 & 1 & 1 \\
$z b p$ & 0 & 0 & 0 & 0 & 0 & 0 & 0 & 0 & 1 & 1 & 1 & 1 \\
\hline
\end{tabular}

It is clear that this relation is a semiorder.

It is easy to check that the traces are as follows.

On attribute 2, we have $a \succ_{2}^{+} b$ and $a \succ_{2}^{-} b$. Hence, $A C 1_{2}, A C 2_{2}$, and $A C 3_{2}$ hold. Since $X_{2}$ has only two elements, $A C 1_{2}^{* *}, A C 2_{2}^{* *}, A C 3_{2}^{* *}$, and $A C 4_{2}^{* *}$ trivially hold.

On attribute 3, we have $q \succ_{3}^{+} p$ and $q \succ_{3}^{-} p$. Hence, $A C 1_{3}, A C 2_{3}$, and $A C 3_{3}$ hold. Since $X_{3}$ has only two elements, $A C 1_{3}^{* *}, A C 2_{3}^{* *}, A C 3_{3}^{* *}$, and $A C 4_{3}^{* *}$ trivially hold.

On attribute $1, \succsim_{1}^{+}$is a clique. Furthermore, we have $x \succ_{1}^{-} y$ and $x \succ_{1}^{-} z$, but neither $y \succsim_{1}^{-} z$ nor $z \succsim_{1}^{-} y$. It easy to check that this implies that $A C 1_{1}$ and $A C 3_{1}$ hold, while $A C 2_{1}$ is violated.

It remains to check that $A C 1_{1}^{* *}, A C 2_{1}^{* *}, A C 3_{1}^{* *}$, and $A C 4_{1}^{* *}$ are satisfied.

Using Lemma 9 , it is easy to check that $A C 1_{1}^{*}, A C 3_{1}^{*}$, and $A C 4_{1}^{*}$ are satisfied. Indeed, since $\succsim_{1}^{+}$is a clique, the premise of $A C 1_{1}^{*}$ and $A C 3_{1}^{*}$ is never satisfied while the conclusion $A C 4_{1}^{*}$ always holds.

Let us check that $A C 2_{1}^{* *}$ holds.

The second premise of $A C 2_{1}^{* *}$ is $d \succsim\left(z_{1}, b_{-1}\right)$. If $z_{1}=x$ then since all elements of $X_{1}$ are below $x$ according to $\succsim_{1}^{-}$, we will have that $d \succsim\left(y_{1}, b_{-i}\right)$, for all $y_{1} \in X_{1}$.

Similarly, the first premise of $A C 2_{1}^{* *}$ is $c \succsim\left(y_{1}, a_{-1}\right)$. If $y_{1}=x$ then, since all elements of $X_{1}$ are below $x$ according to $\succsim_{1}^{-}$, we will have that $c \succsim\left(x_{1}, a_{-1}\right)$, for all $x_{1} \in X_{1}$.

This shows that the only possible violations of $A C 2_{1}^{* *}$ will occur if either $y_{1}=y$ and $z_{1}=z$ or $y_{1}=z$ and $z_{1}=y$. 
Let us consider the first case with $y_{1}=y$ and $z_{1}=z$. The premises of $A C 2_{1}^{* *}$ state that $c \succsim\left(y, a_{-1}\right), d \succsim\left(z, b_{-1}\right)$, and $c \succsim\left(z, a_{-1}\right)$. It is easy to check that whenever we have both $c \succsim\left(y, a_{-1}\right)$ and $c \succsim\left(z, a_{-1}\right)$ we also have $c \succsim\left(x, a_{-1}\right)$. Hence, the first conclusion of $A C 2_{1}^{* *}$ will hold.

Let us now consider the second case in which $y_{1}=z$ and $z_{1}=y$. The premises of $A C 2_{1}^{* *}$ state that $c \succsim\left(z, a_{-1}\right), d \succsim\left(y, b_{-1}\right)$, and $c \succsim\left(y, a_{-1}\right)$. As observed above, whenever we have both $c \succsim\left(z, a_{-1}\right)$ and $c \succsim\left(y, a_{-1}\right)$, we also have that $c \succsim\left(x, a_{-1}\right)$. Hence the first conclusion of $A C 2_{1}^{* *}$ is satisfied.

Hence $A C 2_{1}^{* *}$ holds.

Example 7 . Let $\succsim$ on $X$ be defined by the following table:

\begin{tabular}{ccccccccccccc}
\hline & $x a q$ & $y a q$ & $x a p$ & $x b p$ & $x b q$ & $y a p$ & $y b p$ & $y b q$ & $z a p$ & $z a q$ & $z b p$ & $z b q$ \\
$x a q$ & 1 & 1 & 1 & 1 & 1 & 1 & 1 & 1 & 1 & 1 & 1 & 1 \\
$y a q$ & 1 & 1 & 1 & 1 & 1 & 1 & 1 & 1 & 1 & 1 & 1 & 1 \\
$x a p$ & 1 & 1 & 1 & 1 & 1 & 1 & 1 & 1 & 1 & 1 & 1 & 1 \\
$x b p$ & 1 & 1 & 1 & 1 & 1 & 1 & 1 & 1 & 1 & 1 & 1 & 1 \\
$x b q$ & 1 & 1 & 1 & 1 & 1 & 1 & 1 & 1 & 1 & 1 & 1 & 1 \\
$y a p$ & 1 & 1 & 1 & 1 & 1 & 1 & 1 & 1 & 1 & 1 & 1 & 1 \\
$y b p$ & 1 & 1 & 1 & 1 & 1 & 1 & 1 & 1 & 1 & 1 & 1 & 1 \\
$y b q$ & 1 & 1 & 1 & 1 & 1 & 1 & 1 & 1 & 1 & 1 & 1 & 1 \\
$z a p$ & 1 & 1 & 1 & 1 & 1 & 1 & 1 & 1 & 1 & 1 & 1 & 1 \\
$z a q$ & 1 & 1 & 1 & 1 & 1 & 1 & 1 & 1 & 1 & 1 & 1 & 1 \\
$z b p$ & 1 & 1 & 1 & 1 & 1 & 1 & 1 & 1 & 1 & 1 & 1 & 1 \\
$z b q$ & 0 & 0 & 1 & 1 & 1 & 1 & 1 & 1 & 1 & 1 & 1 & 1 \\
\hline
\end{tabular}

This relation is a semiorder.

It is easy to check that the traces are as follows.

On attribute 2, we have $a \succ_{2}^{+} b$ and $a \succ_{2}^{-} b$. Hence, $A C 1_{2}, A C 2_{2}$, and $A C 3_{2}$ hold. Since $X_{2}$ has only two elements, $A C 1_{2}^{* *}, A C 2_{2}^{* *}, A C 3_{2}^{* *}$, and $A C 4_{2}^{* *}$ trivially hold.

On attribute 1 , we have $\left[x \sim_{1}^{+} y\right] \succ_{1}^{+} z$ and $\left[\begin{array}{lll}x \sim_{1}^{-} & y\end{array}\right] \succ_{1}^{-} z$. Using Lemma 9 , it is easy to check that $A C 1_{1}^{*}, A C 2_{1}^{*}, A C 3_{1}^{*}$, and $A C 4_{1}^{*}$ hold. This shows that $A C 1_{1}, A C 2_{1}, A C 3_{1}, A C 1_{1}^{* *}, A C 2_{1}^{* *}, A C 3_{1}^{* *}$, and $A C 4_{1}^{* *}$ hold.

On attribute 3, we have $p \succ_{3}^{+} q$ and $q \succ_{3}^{-} p$. Hence, $A C 1_{3}$ and $A C 2_{3}$ hold but $A C 3_{3}$ is violated. Since $X_{3}$ has only two elements, $A C 1_{3}^{* *}, A C 2_{3}^{* *}$, $A C 3_{3}^{* *}$, and $A C 4_{3}^{* *}$ trivially hold. 


\section{Proof of Lemma 18}

We have to show that in the class of all weak orders on $X$, conditions $A C 1$ and $A C 1^{* *}$ are independent. We need two examples. The first gives an example of a weak order satisfying $A C 1$ and $A C 1_{i}^{* *}$ and on all but one of the attributes. The second gives an example of a weak order satisfying $A C 1^{* *}$ and $A C 1_{i}$ on all but one of the attributes.

Example 8. Let $\succsim$ on $X$ be the weak order obtained using an additive value function model with the following value functions: $v_{1}(x)=0, v_{1}(y)=1$, $v_{1}(z)=2, v_{2}(a)=0, v_{2}(b)=1, v_{3}(p)=0$, and $v_{3}(q)=1$. It is easy to check that, for this weak order, we have: $z \succ_{1}^{+} y \succ_{1}^{+} x, b \succ_{2}^{+} a$, and $q \succ_{3}^{+} p$. This shows that conditions $A C 1, A C 1_{2}^{* *}$, and $A C 1_{3}^{* *}$ hold. By construction, $A C 1_{1}^{* *}$ is violated.

Example 9. Let $\succsim$ on $X$ be defined by the following table:

\begin{tabular}{ccccccccccccc}
\hline & $x b p$ & $x b q$ & $y b p$ & $y b q$ & $z a p$ & $z b p$ & $z b q$ & $x a p$ & $y a p$ & $y a q$ & $x a q$ & $z a q$ \\
$x b p$ & 1 & 1 & 1 & 1 & 1 & 1 & 1 & 1 & 1 & 1 & 1 & 1 \\
$x b q$ & 1 & 1 & 1 & 1 & 1 & 1 & 1 & 1 & 1 & 1 & 1 & 1 \\
$y b p$ & 1 & 1 & 1 & 1 & 1 & 1 & 1 & 1 & 1 & 1 & 1 & 1 \\
$y b q$ & 1 & 1 & 1 & 1 & 1 & 1 & 1 & 1 & 1 & 1 & 1 & 1 \\
$z a p$ & 1 & 1 & 1 & 1 & 1 & 1 & 1 & 1 & 1 & 1 & 1 & 1 \\
$z b p$ & 1 & 1 & 1 & 1 & 1 & 1 & 1 & 1 & 1 & 1 & 1 & 1 \\
$z b q$ & 1 & 1 & 1 & 1 & 1 & 1 & 1 & 1 & 1 & 1 & 1 & 1 \\
$x a p$ & 0 & 0 & 0 & 0 & 0 & 0 & 0 & 1 & 1 & 1 & 1 & 1 \\
$y a p$ & 0 & 0 & 0 & 0 & 0 & 0 & 0 & 1 & 1 & 1 & 1 & 1 \\
$y a q$ & 0 & 0 & 0 & 0 & 0 & 0 & 0 & 1 & 1 & 1 & 1 & 1 \\
$x a q$ & 0 & 0 & 0 & 0 & 0 & 0 & 0 & 0 & 0 & 0 & 1 & 1 \\
$z a q$ & 0 & 0 & 0 & 0 & 0 & 0 & 0 & 0 & 0 & 0 & 1 & 1 \\
\hline
\end{tabular}

It is clear that this relation is the weak order on $X$ such that:

$[x b p, x b q, y b p, y b q, z a p, z b p, z b q] \succ[x a p, y a p, y a q] \succ[x a q, z a q]$

It is easy to check that we have $b \succ_{2}^{+} a$, and $q \succ_{3}^{+} p$. Using Lemma 9 shows that $A C 1_{i}^{*}$, and, hence, $A C 1_{i}$ and $A C 1_{i}^{* *}$ hold for $i=2,3$.

On attribute 1 , we have $y \succ_{1}^{+} x, z \succ_{1}^{+} x$, but neither $y \succ_{1}^{+} z$ nor $z \succ_{1}^{+} y$, so that $A C 1_{1}$ is violated.

Let us check that $A C 1_{1}^{* *}$ holds. 
The second premise of $A C 1_{1}^{* *}$ is $\left(y_{1}, b_{-1}\right) \succsim d$. If $y_{1}=x$ then, since all elements of $X_{1}$ are above $x$ according to $\succsim_{1}^{+}$, we will have that $\left(z_{1}, b_{-1}\right) \succsim d$, for all $z_{1} \in X_{1}$.

Similarly, the first premise of $A C 1_{1}^{* *}$ is $\left(x_{1}, a_{-1}\right) \succsim c$. If $x_{1}=x$ then, since all elements of $X_{1}$ are above $x$ according to $\succsim_{1}^{+}$, we will have that $\left(y_{1}, a_{-1}\right) \succsim c$, for all $y_{1} \in X_{1}$.

This shows that the only possible violations of $A C 1_{1}^{* *}$ will occur if either $x_{1}=y$ and $y_{1}=z$ or $x_{1}=z$ and $y_{1}=y$.

Let us consider the first case with $x_{1}=y$ and $y_{1}=z$. The premises of $A C 1_{1}^{* *}$ state that $\left(y, a_{-1}\right) \succsim c,\left(z, b_{-1}\right) \succsim d$, and $\left(y, b_{-1}\right) \succsim d$. It is easy to check that whenever we have both $\left(z, b_{-1}\right) \succsim d$ and $\left(y, b_{-1}\right) \succsim d$, we also have that $\left(x, b_{-1}\right) \succsim d$. Hence the second conclusion of $A C 1_{1}^{* *}$ is satisfied.

Let us now consider the second case in which $x_{1}=z$ and $y_{1}=y$. The premises of $A C 1_{1}^{* *}$ state that $\left(z, a_{-1}\right) \succsim c,\left(y, b_{-1}\right) \succsim d$, and $\left(z, b_{-1}\right) \succsim d$. As observed above, whenever we have both $\left(y, b_{-1}\right) \succsim d$ and $\left(z, b_{-1}\right) \succsim d$, we also have that $\left(x, b_{-1}\right) \succsim d$. Hence the second conclusion of $A C 1_{1}^{* *}$ is satisfied.

Hence $A C 1_{1}^{* *}$ holds.

\section{Multiple reference points}

\section{D.1. Definition}

The model defined below, building on Rolland (2003, 2006a,b, 2008, 2011), extends Definition 1 to deal with the case of multiple reference points. In this model, there is a semiorder $R_{i}$ on each attribute. In order to compare the alternatives $x$ and $y$, we first compare each of them to a number of "reference points" $\pi^{1}, \pi^{2}, \ldots, \pi^{\ell}$ only using "ordinal considerations". For each profile $\pi^{k}$, we compute the subsets of attributes $\mathcal{R}^{k}(x)=\left\{i \in N: x_{i} R_{i} \pi_{i}^{k}\right\}$ and $\mathcal{R}^{k}(y)=\left\{i \in N: y_{i} R_{i} \pi_{i}^{k}\right\}$. The comparison of $x$ and $y$ is based on the two $\ell$-tuples $\left(\mathcal{R}^{1}(x), \mathcal{R}^{2}(x), \ldots, \mathcal{R}^{\ell}(x)\right)$ and $\left(\mathcal{R}^{1}(y), \mathcal{R}^{2}(y), \ldots, \mathcal{R}^{\ell}(y)\right)$. This comparison uses an "importance relation" that will only be required to be monotonic w.r.t. inclusion.

Definition 12. A binary relation $\succsim$ is a Relation with Multiple Reference Points (or more briefly is a RMRP) if:

- for all $i \in N$, there is a semiorder $R_{i}$ on $X_{i}$,

- there are $\ell \in \mathbb{N}^{+}$elements of $X, \pi^{1}, \pi^{2}, \ldots, \pi^{\ell}$, interpreted as $\ell$ "reference points", 
- there is a binary relation $\unrhd_{L}$ on $\left(2^{N}\right)^{\ell}$ that is monotonic w.r.t. inclusion, i.e., for all $A^{1}, B^{1}, C^{1}, D^{1}, \ldots, A^{\ell}, B^{\ell}, C^{\ell}, D^{\ell} \subseteq N$,

$$
\left(A^{1}, \ldots, A^{\ell}\right) \unrhd_{L}\left(B^{1}, \ldots, B^{\ell}\right) \Rightarrow\left(C^{1}, \ldots, C^{\ell}\right) \unrhd_{L}\left(D^{1}, \ldots, D^{\ell}\right),
$$

whenever, for all $k \in L=\{1,2, \ldots, \ell\}, C^{k} \supseteq A^{k}, B^{k} \supseteq D^{k}$, and there are $x, y, z, w \in X$ such that $\mathcal{R}^{L}(x)=\left(A^{1}, \ldots, A^{\ell}\right), \mathcal{R}^{L}(y)=$ $\left(B^{1}, \ldots, B^{\ell}\right), \mathcal{R}^{L}(z)=\left(C^{1}, \ldots, C^{\ell}\right)$, and $\mathcal{R}^{L}(w)=\left(D^{1}, \ldots, D^{\ell}\right)$,

such that, for all $x, y \in X$,

$$
x \succsim y \Leftrightarrow\left(\mathcal{R}^{1}(x), \mathcal{R}^{2}(x), \ldots, \mathcal{R}^{\ell}(x)\right) \unrhd_{L}\left(\mathcal{R}^{1}(y), \mathcal{R}^{2}(y), \ldots, \mathcal{R}^{\ell}(y)\right),
$$

where $\mathcal{R}^{k}(x)=\left\{i \in N: x_{i} R_{i} \pi_{i}^{k}\right\}$ and $\mathcal{R}^{L}(x)=\left(\mathcal{R}^{1}(x), \mathcal{R}^{2}(x), \ldots, \mathcal{R}^{\ell}(x)\right)$.

We write $\mathcal{R}^{L}(x) \sqsupseteq \mathcal{R}^{L}(y)$ to mean that, for all $k \in L, \mathcal{R}^{k}(x) \supseteq \mathcal{R}^{k}(y)$.

\section{D.2. Results}

We start by showing that a RMRP is a particular case of model (M).

Lemma 20. If a binary relation on $X$ is a RMRP with $\ell$ reference points, it satisfies $A C 1, A C 2$ and $A C 3$, so that all relations $\succsim_{i}^{ \pm}$are weak orders. Furthermore, for all $i \in N$, the weak order $\succsim_{i}^{ \pm}$has at most $\ell+1$ distinct equivalence classes.

Proof. Let us show that $A C 1$ holds. Suppose that $\left(x_{i}, a_{-i}\right) \succsim c$ and $\left(y_{i}, b_{-i}\right) \succsim$ $d$. Because $R_{i}$ is a semiorder, we know (see the end of Section 2.1) that the relation $R_{i}^{\circ}$ is a weak order. By construction, we have either $x_{i} R_{i}^{\circ} y_{i}$ or $y_{i} R_{i}^{\circ} x_{i}$.

Suppose that $x_{i} R_{i}^{\circ} y_{i}$. By construction, for all $k \in L, y_{i} R_{i} p_{i}^{k}$ implies $x_{i} R_{i} p_{i}^{k}$. This implies $\mathcal{R}^{L}\left(\left(x_{i}, b_{-i}\right)\right) \sqsupseteq \mathcal{R}^{L}\left(\left(y_{i}, b_{-i}\right)\right)$. Since $\left(y_{i}, b_{-i}\right) \succsim d$, we know that $\mathcal{R}^{L}\left(\left(y_{i}, b_{-i}\right)\right) \unrhd_{L} \mathcal{R}^{L}(d)$. Using the fact that $\unrhd_{L}$ is monotonic w.r.t. inclusion, we obtain $\mathcal{R}^{L}\left(\left(x_{i}, b_{-i}\right)\right) \unrhd_{L} \mathcal{R}^{L}(d)$, so that $\left(x_{i}, b_{-i}\right) \succsim d$. A similar proof shows that if $y_{i} R_{i}^{\circ} x_{i}$ then we have $\left(y_{i}, a_{-i}\right) \succsim c$. Hence, $A C 1$ holds.

The proof for $A C 2$ and $A C 3$ is similar.

Let us show that the weak order $\succsim_{i}^{ \pm}$has at most $\ell+1$ distinct equivalence classes. For all $k \in L$, define $\mathcal{A}_{i}^{k}=\left\{x_{i} \in X_{i}: x_{i} R_{i} \pi_{i}^{k}\right\}$. Because $R_{i}$ is a semiorder, the relation $R_{i}^{\circ}$ is a weak order. Let $k, k^{\prime} \in L$. We have either $\pi_{i}^{k} R_{i}^{\circ} \pi_{i}^{k^{\prime}}$ or $\pi_{i}^{k^{\prime}} R_{i}^{\circ} \pi_{i}^{k}$. Hence, we have either $\mathcal{A}_{i}^{k} \subseteq \mathcal{A}_{i}^{k^{\prime}}$ or $\mathcal{A}_{i}^{k^{\prime}} \subseteq \mathcal{A}_{i}^{k}$. This 
shows that, for all $i \in N$, the sets $\mathcal{A}_{i}^{k}, k \in L$ are nested. Moreover, it is clear that if $x_{i}, y_{i} \in X_{i}$ belong exactly to the same subsets $\mathcal{A}_{i}^{k}, k \in L$, we must have $x_{i} \sim_{i}^{ \pm} y_{i}$. This completes the proof since the sets $\mathcal{A}_{i}^{k}$ are nested.

The above lemma shows that a RMRP satisfies $A C 1, A C 2$ and $A C 3$ and is such that, for all $i \in N, X_{i} / \sim_{i}^{ \pm}$is finite. Hence, it has a representation in model (M). We show below that the converse is true, as soon as all sets $X_{i} / \sim_{i}^{ \pm}$are finite.

Lemma 21. Let $\succsim$ be a binary relation on $X$. If $\succsim$ satisfies $A C 1, A C 2$, and $A C 3$ and, for all $i \in N$, the sets $X_{i} / \sim_{i}^{ \pm}$are finite then $\succsim$ is a $R M R P$.

Proof. For all $i \in N$, we know that $\succsim_{i}^{ \pm}$is a weak order and that $X_{i} / \sim_{i}^{ \pm}$is finite. Let $\ell_{i}$ be the number of distinct equivalence classes of $X_{i} / \sim_{i}^{ \pm}$. Let $\ell=\max _{i \in N} \ell_{i}$. The proof will be complete if we show that $\succsim$ is a RMRP.

By construction, there at least one $i \in N$ such that $\succsim_{i}^{ \pm}$has exactly $\ell$ distinct equivalence classes. On all such attributes $i \in N$, we define $E_{i}^{k}$, for $k=1,2, \ldots, \ell$, as the set containing all elements in the $k$ th equivalence class of $\succsim_{i}^{ \pm}$. Consider now an attribute $j \in N$ such that $\succsim_{j}^{ \pm}$has $\ell_{j}$ distinct equivalence classes with $\ell_{j}<\ell$. For all such attributes $j \in N$, we define $E_{j}^{k}$, for $k=1,2, \ldots, \ell_{j}$, as the set containing all elements in the $k$ th equivalence class of $\succsim_{j}^{ \pm}$. For $k=\ell_{j}+1, \ell_{j}+2, \ldots, \ell$, we define $E_{j}^{k}=E_{j}^{\ell_{j}}$.

For all $i \in N$, let us take $R_{i}=\succsim_{i}^{ \pm}$.

We use $\ell$ profiles $\pi^{1}, \pi^{2}, \ldots, \pi^{\ell}$ that are build as follows. For all $i \in N$ and $k \in L$, let $\pi_{i}^{k}$ be any element belonging to $E_{i}^{\ell-k+1}$.

We define the binary relation $\unrhd_{L}$ on $\left(2^{N}\right)^{\ell}$ letting $\left(A^{1}, \ldots, A^{\ell}\right) \unrhd_{L}\left(B^{1}, \ldots, B^{\ell}\right)$ whenever there are $x, y \in X$ such that $x \succsim y, \mathcal{R}^{L}(x)=\left(A^{1}, \ldots, A^{\ell}\right)$, and $\mathcal{R}^{L}(y)=\left(B^{1}, \ldots, B^{\ell}\right)$.

We claim that the family of reference points $\pi^{1}, \pi^{2}, \ldots, \pi^{\ell}$ together with the weak orders $R_{i}=\succsim_{i}^{ \pm}$and the relation $\unrhd_{L}$ is a representation of $\succsim$ in model (RMRP).

Suppose first that $x \succsim y$. Then, by construction, we have $\mathcal{R}^{L}(x) \unrhd_{L}$ $\mathcal{R}^{L}(y)$.

Suppose now that $\left(A^{1}, \ldots, A^{\ell}\right) \unrhd_{L}\left(B^{1}, \ldots, B^{\ell}\right)$ and consider $x, y \in$ $X$ such that $\mathcal{R}^{L}(x)=\left(A^{1}, \ldots, A^{\ell}\right)$, and $\mathcal{R}^{L}(y)=\left(B^{1}, \ldots, B^{\ell}\right)$. Because $\left(A^{1}, \ldots, A^{\ell}\right) \unrhd_{L}\left(B^{1}, \ldots, B^{\ell}\right)$, we know that there are $z, w \in X$ such that $\mathcal{R}^{L}(z)=\left(A^{1}, \ldots, A^{\ell}\right), \mathcal{R}^{L}(w)=\left(B^{1}, \ldots, B^{\ell}\right)$, and $z \succsim w$. Given the construction of the profiles $\pi^{1}, \pi^{2}, \ldots, \pi^{\ell}, \mathcal{R}^{L}(x)=\mathcal{R}^{L}(z)$ implies that, for all 
$i \in N, x_{i} \sim_{i}^{ \pm} z_{i}$. Similarly, $\mathcal{R}^{L}(y)=\mathcal{R}^{L}(w)$ implies that, for all $i \in N$, $y_{i} \sim_{i}^{ \pm} w_{i}$. Using (4), we obtain $x \succsim y$.

It remains to show that $\unrhd_{L}$ is monotonic w.r.t. inclusion.

Suppose that $\left(A^{1}, \ldots, A^{\ell}\right) \unrhd_{L}\left(B^{1}, \ldots, B^{\ell}\right)$. This implies that $x \succsim y$, for some $x, y \in X$ such that $\mathcal{R}^{L}(x)=\left(A^{1}, \ldots, A^{\ell}\right)$, and $\mathcal{R}^{L}(y)=\left(B^{1}, \ldots, B^{\ell}\right)$. Take any $\left(C^{1}, \ldots, C^{\ell}\right)$ and $\left(D^{1}, \ldots, D^{\ell}\right)$ such that, $k \in L, C^{k} \supseteq A^{k}, B^{k} \supseteq D^{k}$, and there are $z, w \in X$ such that $\mathcal{R}^{L}(z)=\left(C^{1}, \ldots, C^{\ell}\right)$, and $\mathcal{R}^{L}(w)=$ $\left(D^{1}, \ldots, D^{\ell}\right)$. By construction of the family of reference points $\pi^{1}, \pi^{2}, \ldots, \pi^{\ell}$, this implies that $z_{i} \succsim_{i}^{ \pm} x_{i}$ and $y_{i} \succsim_{i}^{ \pm} w_{i}$, for all $i \in N$. Using, (3), we obtain $z \succsim w$, so that $\left(C^{1}, \ldots, C^{\ell}\right) \unrhd_{L}\left(D^{1}, \ldots, D^{\ell}\right)$, as required.

Lemmas 20 and 21 show that, whenever $X_{i} / \sim_{i}^{ \pm}$is finite, for all $i \in N$, the Model with Multiple Points of Reference is equivalent to model (M). Moreover:

- if $\succsim$ has a representation in the Model with Multiple Points of Reference, it also has a representation in the Model with Multiple Points of Reference in which all relations $R_{i}$ are weak orders,

- if $\succsim$ has a representation in the Model with Multiple Points of Reference, it also has a representation in which reference points dominates each other according to the weak orders $R_{i}$, i.e., for all $k \in\{2,3, \ldots, \ell\}$ and all $i \in N, \pi_{i}^{k} R_{i} \pi_{i}^{k-1}$. In such a representation, for all $x \in X$, with $\mathcal{R}^{L}(x)=\left(A^{1}, \ldots, A^{\ell}\right)$ we have $A^{1} \supseteq A^{2} \supseteq \cdots \supseteq A^{\ell}$ (this was already observed in Rolland 2008, p. 51).

Indeed, the reference points $\pi^{1}, \pi^{2}, \ldots, \pi^{\ell}$ and the relations $R_{i}$ that are built in the proof of Lemma 21 have these two properties.

Our findings are summarized below.

Theorem 3. A binary relation $\succsim$ on $X$ is a RMRP iff it satisfies $A C 1, A C 2$ and $A C 3$ and, for all $i \in N$, the set $X_{i} / \sim_{i}^{ \pm}$is finite.

If a relation $\succsim$ is a $R M R P$, it always has a representation in which all relations $R_{i}$ are weak orders and in which the $\ell$ points of reference are such that for all $k \in\{2,3, \ldots, \ell\}$ and all $i \in N, \pi_{i}^{k} R_{i} \pi_{i}^{k-1}$.

The uniqueness of the representation of RMRP is obviously quite weak. Since its study is cumbersome and does not appear to be particularly informative, we do not detail this point. 
The above result shows that in a reflexive RMRP all relations $\succsim_{i}^{ \pm}$have a finite number of equivalence classes. We have seen above (see the proof of Lemma 15) that there are CR-AT in which the relations $\succsim_{i}^{ \pm}$have infinitely many equivalence classes. Hence, there are CR-AT that are not reflexive RMRP. The converse is also true since a reflexive RMRP may fail to independent whereas we know that a CR-AT always is. The claim of Rolland (2003, 2006a, 2008) that models using reference points are more "flexible" than concordance relations is nevertheless clearly true if attention is restricted to the case of finite sets $X$. Indeed, a CR-AT always satisfies conditions $A C 1$, $A C 2$ and $A C 3$. Moreover, on finite sets, the condition stating that, for all $i \in N$, the set $X_{i} / \sim_{i}^{ \pm}$is finite trivially holds. Hence, in this particular but important case, all CR-AT are reflexive RMRP.

Using Lemma 5, it is easy to formulate a result characterizing model (RMRP) when $\succsim$ is a weak order. In this case, we know that the three conditions $A C 1$, $A C 2$ and $A C 3$ are equivalent and may be replaced by weak separability. For the record, we state the following:

Proposition 5. Let $\succsim$ be a weak order on $X$. It is a Relation with Multiple Reference Points iff it satisfies $A C 1$ and, for all $i \in N$, the set $X_{i} / \sim_{i}^{ \pm}$is finite.

Since the relation $\unrhd_{L}$ is defined on the finite set $\left(2^{N}\right)^{\ell}$, it is simple to show that a RMRP that is a weak order can only have a finite number of equivalence classes.

Consider now a relation $\succsim$ defined on a finite set $X$. It will be a RMRP as soon as it satisfies $A C 1$. In view of Proposition 4 , it will have a representation in the discrete Sugeno integral model if, furthermore, it is 2-graded. Clearly, a relation satisfying $A C 1$ does not have to be 2 -graded. This shows that, contrary to the situation with RSRP, a RMRP may not have a representation in the discrete Sugeno integral model. This was already noted in Rolland (2008, Example 61, p. 138).

\section{References}

F. Aleskerov, D. Bouyssou, and B. Monjardet. Utility maximization, choice and preference. Springer-Verlag, Berlin, 2nd edition, 2007.

D. Bouyssou and T. Marchant. An axiomatic approach to noncompensatory sorting methods in MCDM, I: The case of two categories. European Journal of Operational Research, 178(1):217-245, 2007a. 
D. Bouyssou and T. Marchant. An axiomatic approach to noncompensatory sorting methods in MCDM, II: More than two categories. European Journal of Operational Research, 178(1):246-276, 2007b.

D. Bouyssou and M. Pirlot. Nontransitive decomposable conjoint measurement. Journal of Mathematical Psychology, 46(6):677-703, 2002.

D. Bouyssou and M. Pirlot. 'Additive difference' models without additivity and subtractivity. Journal of Mathematical Psychology, 48(4):263-291, 2004a.

D. Bouyssou and M. Pirlot. Preferences for multiattributed alternatives: Traces, dominance, and numerical representations. Journal of Mathematical Psychology, 48(3):167-185, 2004b.

D. Bouyssou and M. Pirlot. Following the traces: An introduction to conjoint measurement without transitivity and additivity. European Journal of Operational Research, 163(2):287-337, 2005a.

D. Bouyssou and M. Pirlot. A characterization of concordance relations. European Journal of Operational Research, 167(2):427-443, 2005b.

D. Bouyssou and M. Pirlot. Further results on concordance relations. European Journal of Operational Research, 181(1):505-514, 2007.

D. Bouyssou, T. Marchant, and M. Pirlot. A conjoint measurement approach to the discrete Sugeno integral. In S. Brams, W. V. Gehrlein, and F. S. Roberts, editors, The Mathematics of Preference, Choice and Order. Essays in Honor of Peter C. Fishburn, pages 85-109. Springer, 2009.

D. Dubois, H. Fargier, and P. Perny. Qualitative decision theory with preference relations and comparative uncertainty: An axiomatic approach. Artificial Intelligence, 148(1-2):219-260, 2003. Corrigendum: Artificial Intelligence, Volume 171(5-6), 2007, 361-362.

H. Fargier and P. Perny. Qualitative decision models under uncertainty without the commensurability assumption. In K. B. Laskey and H. Prade, editors, Proceedings of Uncertainty in Artificial Intelligence, pages 188-195. Morgan Kaufmann Publishers, 1999. 
S. Greco, B. Matarazzo, and R. Słowiński. Axiomatic characterization of a general utility function and its particular cases in terms of conjoint measurement and rough-set decision rules. European Journal of Operational Research, 158(2):271-292, 2004.

D. Kahneman and A. Tversky. Prospect theory: An analysis of decision under risk. Econometrica, 47(2):263-291, 1979.

P. Perny and A. Rolland. Reference-dependent qualtitative models for decision making under uncertainty. In G. Brewka, S. Coradeschi, A. Perini, and P. Traverso, editors, Proceedings ECAI-2006, 20th European Conference on Artificial Intelligence, pages 422-426. IOS Press, 2006.

A. Rolland. Agrégation de préférences et point de référence. In Actes de l'école d'automne de Recherche Opérationnelle, pages 154-160. Université de Tours, 2003.

A. Rolland. Points de référence en décision multicritère. In Actes de la Yème Conférence ROADEF, pages 153-167. Presses Universitaires de Valenciennes, 2006a.

A. Rolland. On bi-capacity-based concordance rules in multicriteria decision making. In B. Bouchon-Meunier, G. Coletti, and R. R. Yager, editors, Modern Information Processing: From theory to applications, pages 231245. Elsevier, 2006b.

A. Rolland. Procédures d'agrégation ordinale de préférences avec points de référence pour l'aide à la décision. Thèse de doctorat, Université Pierre et Marie Curie, Paris, 2008. Available from http://eric.univ-lyon2.fr/ arolland/index $\cdot$ php? choix=3.

A. Rolland. Reference-based preferences aggregation procedures in multicriteria decision making. Working Paper, Laboratoire ERIC, Université Lumière Lyon 2, April 2011.

B. Roy and D. Bouyssou. Aide multicritère à la décision : méthodes et cas. Economica, Paris, 1993.

A. Tversky and D. Kahneman. Rational choice and the framing of decisions. Journal of Business, 59(4):S251-S278, 1986. 
A. Tversky and D. Kahneman. Advances in prospect theory: Cumulative representation of uncertainty. Journal of Risk and Uncertainty, 5(4):297323, 1992. 